Published in final edited form as:

Nat Biomed Eng. 2018 May ; 2(5): 326-337. doi:10.1038/s41551-018-0214-1.

\title{
A ribonucleoprotein octamer for targeted siRNA delivery
}

\author{
Wanyi Tai ${ }^{1}$, Junwei $\mathrm{Li}^{1}$, Eva Corey ${ }^{2}$, Xiaohu Gao ${ }^{1,}$, \\ ${ }^{1}$ Department of Bioengineering, University of Washington, Seattle, WA 98195, USA \\ ${ }^{2}$ Department of Urology, University of Washington, Seattle, WA 98195, USA
}

\section{Abstract}

Hurdles in cell-specific delivery of small interfering RNA (siRNA) in vivo hinder the clinical translation of RNA interference (RNAi). A fundamental problem concerns conflicting requirements for the design of the delivery vehicles: cationic materials facilitate cargo condensation and endosomolysis, yet hinder in vivo targeting and colloidal stability. Here, we describe a self-assembled, compact $(\sim 30 \mathrm{~nm})$ and biocompatible ribonucleoprotein-octamer nanoparticle that achieves endosomal destabilization and targeted delivery. The protein octamer consists of a poly(ethylene glycol) scaffold, a sterically masked endosomolytic peptide, and a double-stranded RNA-binding domain, provides a discrete number of siRNA loading sites and a high siRNA payload (> $30 \mathrm{wt} \%$ ), and offers flexibility in both siRNA and targeting-ligand selection. We show that a ribonucleoprotein octamer against the polo-like kinase 1 (Plk1) gene and bearing a ligand that binds to prostate specific membrane antigen (PSMA) leads to efficient gene silencing in prostate tumour cells in vitro and when intravenously injected in mouse models of prostate cancer. The octamer's versatile nanocarrier design should offer opportunities for the clinical translation of therapies based on intracellularly acting biologics.

\begin{abstract}
Short interference RNA (siRNA) is of considerable interest in biology and medicine because of its unique capability in probing gene functions and in attenuating progression of some currently incurable diseases. ${ }^{1}$ However, similar to other antisense therapeutics, in vivo use of siRNA represents a major challenge due to the delivery problem. ${ }^{2}$ This problem could also potentially propagate into in vivo applications of the genome editing technology $\mathrm{y}^{3-6}$. Advances in bioengineering and nanotechnology in the past several decades have produced a number of delivery technologies, including liposome, polymers, peptides, and inorganic nanoparticles. ${ }^{7-9}$ Their clinical translation and commercialization, however, are still limited to delivery of siRNA to the liver. ${ }^{10-11}$ Targeted systemic delivery of biologics to other organs and cells (in particular, specific cell compartments) of interest remains a major problem.
\end{abstract}

\footnotetext{
Users may view, print, copy, and download text and data-mine the content in such documents, for the purposes of academic research, subject always to the full Conditions of use: http://www.nature.com/authors/editorial_policies/license.html\#terms

"Correspondence should be addressed to X.H.G. (xgao@uw.edu).

AUTHOR CONTRIBUTIONS

W.Y.T. and X.H.G. conceived the idea and designed the project. W.Y.T. and J.W.L. performed the experiments. W.Y.T., E.C. and X.H.G. analyzed the data and wrote the paper.

COMPETING INTERESTS

The authors declare no competing financial and non-financial interests.
} 
A fundamental issue of in vivo targeted siRNA delivery is the conflicting design requirements during different stages of siRNA interaction with the biological system. As siRNA molecules are large in size, hydrophilic, and susceptible to enzymatic degradation, delivery vehicles are needed to bring them to desired cell types and intracellular spaces. During the initial step of administration and blood circulation, the delivery vehicle-siRNA complex should avoid excessive positive charges to reduce toxicity, aggregation in serum, and non-specific binding with serum proteins and cells. At the same time, because siRNA molecules are negatively charged, the delivery vehicles need to be highly positively charged for siRNA condensation and protection. Furthermore, during the stage of endosome escape (after siRNAs are endocytosed), cationic materials are again needed to facilitate endosomolysis. Indeed, regardless of the chemical composition (e.g., lipids, polymers, and inorganic materials), vast majority of siRNA delivery vehicles are positive charged. ${ }^{12-13}$ As a result of the contradictory requirements to avoid positive charges for in vivo targeting and to have positive charges for cargo condensation and endosome escape, the conventional delivery vehicles are highly efficient in transfecting siRNA into cells in vitro, but have largely failed the task of targeted delivery in vivo. ${ }^{10}$

Here, we report the development towards a 'perfect' siRNA delivery system that simultaneously accommodates all the design requirements, including compact size, precisely controlled siRNA loading, endosomolytic capability, absence of excessive positive charge, immunocompatibility, and flexibility in siRNA and ligand selection. This class of nanocarrier is enabled by two design concepts: i) engineering natural proteins and their assembly in a one-domain-one-function architecture; and ii) sterically masking unnatural functional peptides for immunocompatibility. We demonstrate this idea by combining three key building blocks into a compact nanoparticle with a discrete number of RNA loading sites. The building blocks include a natural human protein, double-strand RNA binding domain (dsRBD), that is capable of binding with double stranded RNA (dsRNA) in a sequence-independent fashion, ${ }^{14}$ a short peptide $\left(\mathrm{H}_{2} \mathrm{E}, 9\right.$ repeats) capable of proton buffering to aid endosomolysis, and a branched polyethylene glycol (PEG) as the structural scaffold. As shown in Figure 1a, upon incubation with a siRNA-targeting ligand bioconjugate (e.g., siRNA-aptamer, siRNA-small molecule ligand, or siRNA-antibody), the siRNA end will dock into the dsRBD domain stoichiometrically, rendering the ribonucleoprotein complex octamer $\left(\mathrm{RNP}_{8}\right)$ complete in desired functionalities, such as high payload of siRNA, exposed and accessible targeting ligand, free of excessive positive surface charges and aggregation, and small in size. More importantly, we took immunocompatibility into consideration during the design for potential clinical translation. The exquisite structure of $\mathrm{RNP}_{8}$ features a protein shell of human origin and a short non-natural peptide sandwiched between two bulky macromolecules (dsRBD and branched PEG). In this arrangement, the endosomolytic $\left(\mathrm{H}_{2} \mathrm{E}\right)_{9}$ peptide is sterically hindered from interacting with macrobiomolecules such as antibodies (typical dimensions of IgG are approximately $14.5 \times$ $8.5 \times 4.0 \mathrm{~nm}),{ }^{15}$ yet still accessible to proton $(\sim 1 \AA)$. 


\section{RESULTS}

\section{Construction and assembly of $\mathrm{RNP}_{\mathbf{8}}$}

Human dsRBD is genetically modified by inserting the endosomolytic $\left(\mathrm{H}_{2} \mathrm{E}\right)_{9}$ peptide to its $\mathrm{C}$-terminus. The $\mathrm{C}$-terminus of the dual functional protein is then activated by Sortase A to introduce an azide group and linked to the branched PEG ( 8 arms) via site-specific click chemistry (Supplementary Figure S1). To saturate the eight reactive sites in the branched PEG, excess dsRBD (with the azide modification) was added. As shown in the electrophoretic mobility shift assay (Figure 1b), higher molar ratio of dsRBD to PEG promotes formation of multivalent structures. When dsRBD reaches 2.5 fold of the stoichiometric ratio to the PEG reactive sites, the desired octamer forms without impurities of lower valence. Excess dsRBD monomer can be easily removed using size-exclusion chromatography (Figure 1c).

In parallel, a bioconjugate was constructed by linking a siRNA sequence of interest (cargo molecule) with a targeting ligand for targeted siRNA delivery. Due to the broad selection of siRNA sequences and targeting ligands (e.g., antibodies, aptamers, and smallmolecules), the combinations are virtually unlimited, potentially enabling precision therapy. For example, several siRNA-aptamer conjugates have been recently used to suppress tumor growth or sensitize tumors for chemo- or radio-therapy, ${ }^{16-18}$ while others were demonstrated to reduce HIV viral loads and protect from helper CD4+ T cell decline in humanized mice. ${ }^{19}$ In our study, we chose a siRNA targeting Plk1, whose depletion induces apoptosis, and a small-molecule ligand, 2-[3-(1,3-dicarboxypropyl)ureido]pentanedioic acid (DUPA), against prostate specific membrane antigen (PSMA) ${ }^{20}$ Previously, PSMA has been identified as one of the most attractive cell surface marker for both prostate cancer cells and neovascular endothelial cells. ${ }^{21}$ Accumulation and retention of PSMA targeting probes at the site of tumor growth is the basis of radioimmuno-scintigraphic scanning and targeted therapy for human prostate cancer metastasis. ${ }^{22}$ DUPA was linked to the $5^{\prime}$ end of the siRNA sense strand with a short PEG spacer in between to provide flexibility to the DUPA ligand (Supplementary Figure S2). To ensure siRNA loading into the RNA-induced silencing complex (RISC), a cleavable disulfide bond was also introduced just before the siRNA strand to allow it to shed the targeting ligand once inside cells (Supplementary Figure S3).

When the dsRBD octamer and the siRNA-DUPA conjugate are mixed together, the siRNA end of the conjugate docks into the peripheral dsRBD in a one-on-one fashion (one dsRBD monomer-one siRNA), whereas the DUPA end remains exposed and accessible. ${ }^{23-25}$ Because the assembly is not based on electrostatic interactions (aggregation free), the ribonucleoprotein octamer is in excellent size range for blood vessel extravasation and deep tissue penetration. ${ }^{26}$ To confirm the functionality of dsRBD after immobilized onto the PEG scaffold, it was incubated with siRNA-DUPA at various molar ratios. As shown in Figure 1d and Supplementary Figure S4, when the siRNA-DUPA/dsRBD octamer ratio is greater than 8 , two discrete bands representing free siRNA-DUPA and saturated $\mathrm{RNP}_{8}$, can be visualized by gel electrophoresis. When the ratio decreases $\left(\mathrm{RNP}_{8}\right.$ becomes less and less saturated), the free siRNA band disappears whereas the unsaturated dsRBD octamer band shifts towards the loading wells (fewer RNA per particle leads to lower surface negative charge and 
consequently slower mobility). This study shows the preserved functionality of dsRBD on the PEG core. Furthermore, the binding stability of $\mathrm{RNP}_{8}$ (between siRNA and dsRBD) was tested in mouse serum. As shown in Figure 1e, the protein-siRNA complex dissociates very slowly, with a half-life exceeding 18 hours. Considering the non-covalent nature of $\mathrm{RNP}_{8}$ self-assembly, this outstanding binding stability pave the road for downstream in vitro and in vivo applications.

Besides gel electrophoresis, we also characterized the fully assembled $\mathrm{RNP}_{8}$ with transmission electron microscopy (TEM). Due to the 3-D structure and the low electron density of the biomolecule nanoparticle, direct observation of its eight arms is difficult. Nevertheless, the TEM micrographs clearly show the uniform size of $\mathrm{RNP}_{8}$ (Figure $1 \mathrm{f}$ \& g). To further confirm the nanoparticle size in solution, dynamic light scattering (DLS) was used to measure the hydrodynamic sizes of the dsRBD octamer (without cargo) and $\mathrm{RNP}_{8}$ (with cargo). As shown in Supplementary Figure S6, the nanocarrier features a size of approximately $29 \mathrm{~nm}$ in diameter, and it increases by $\sim 2.5 \mathrm{~nm}$ after siRNA loading. Zeta potential analysis shows that the surface charges of the nanostructures are $-3.7 \mathrm{mV}$ before cargo loading and $-12.8 \mathrm{mV}$ after siRNA loading, proving $\mathrm{RNP}_{8}$ does not belong to the conventional cationic nanocarriers.

\section{In vitro cell binding and uptake}

To evaluate the cellular binding efficiency, $\mathrm{RNP}_{8}$ was incubated with prostate tumor cells with differential levels of PSMA expression (LNCaP, 22RV1 and PC-3) at room temperature $\left(20^{\circ} \mathrm{C}\right)$ for 1 hour, and the results were benchmarked against siRNA-DUPA conjugate (without the nanocarrier). For quantitative comparison using flow cytometry, the siRNA antisense strand was fluorescently labeled with Alexa Fluor (AF) 555 at the $5^{\prime}$ end (dyes labeling doesn't interference $\mathrm{RNP}_{8}$ assembly, Supplementary Figure S7). A number of interesting results can be observed in Figure 2a. First, both siRNA-DUPA and $\mathrm{RNP}_{8}$ efficiently bind to LNCaP and 22RV1 cells (highly positive in PSMA expression) but not the PC-3 cells (no PSMA expression). This correlation between single cell fluorescence intensity and PSMA expression level demonstrate the binding specificity of the DUPA ligand. To further confirm the specificity of binding, an inhibition assay was conducted utilizing a well-established PSMA inhibitor that shares the same binding pocket with DUPA. ${ }^{20}$ The cell fluorescence signals due to binding with the $\mathrm{RNP}_{8}$ or siRNA-DUPA gradually decreased to the background level with increasing concentrations of the inhibitor (Figure 2b).

Second, when more quantitative comparison was made by plotting cell binding affinity profiles for siRNA-DUPA, $\mathrm{RNP}_{8}$, siRNA-DUPA complexed with a single dsRBD (Figure $2 \mathrm{c}$ ), it was interesting to note that cell binding of $\mathrm{RNP}_{8}$ is approximately 20 -fold higher than that of siRNA-DUPA. This is somewhat counterintuitive since siRNA-DUPA bound to a large dsRBD octamer should have lower binding affinity to PSMA receptors (due to potential steric hindrance from dsRBD) compared to siRNA-DUPA alone. Indeed, when RNP monomer (siRNA-DUPA and dsRBD complex without oligomerizing on the 8-arm PEG) was introduced to the PSMA-positive cells, the cell fluorescence intensity was approximately 2-3 folds lower than that of cells treated with siRNA-DUPA. Therefore, 
the enhanced binding activity of $\mathrm{RNP}_{8}$ is likely due to the multivalency effect. It is known that binding between two entities through multiple molecular recognition events can convert low-affinity monovalent binding to high affinity. ${ }^{27-28}$ To evaluate the multivalency effect for $\mathrm{RNP}_{8}$, the dissociation constant $\left(\mathrm{K}_{\mathrm{D}}\right)$ for $\mathrm{RNP}_{8}$-PSMA receptor binding was measured and compared to siRNA-DUPA binding with PSMA receptor using the surface plasmon resonance (SPR) assay (Figure $2 \mathrm{~d}$ ). $\mathrm{RNP}_{8}$ shows a $\mathrm{K}_{\mathrm{D}}$ of sub-nM, approximately 100 times stronger than that of siRNA-DUPA $\left(\mathrm{K}_{\mathrm{D}}=82.1 \mathrm{nM}\right)$.

Next, we evaluated intracellular uptake by incubating $\mathrm{RNP}_{8}$ with cells at $37^{\circ} \mathrm{C}$ for different durations. After $2 \mathrm{~h}$ incubation, quantitative analysis measuring internalized siRNA using stem-loop polymerase chain reaction (PCR) reveals that both $\mathrm{RNP}_{8}$ and siRNA-DUPA efficiently enter PSMA-positive LNCaP cell, but not the PSMA-negative PC-3 cells. Similar to the cell binding experiment, cell uptake of $\mathrm{RNP}_{8}$ was 6-fold higher than siRNA-DUPA and 30-fold higher than naked siRNA without the DUPA ligand (Figure 3a). The elevated cell uptake of $\mathrm{RNP}_{8}$ is likely resulted from its high binding affinity to cell surface receptors and high payload (each binding event triggers the uptake of 8 siRNA molecules). Furthermore, this uptake is both time- and dose-dependent, and increasing the incubation time or $\mathrm{RNP}_{8}$ concentration leads to higher concentration of siRNA inside cells (Figure 3b).

To follow RNP8's intracellular trafficking, cells were imaged with confocal microscopy. $\mathrm{RNP}_{8}$ particles are mainly associated with the cell membrane after 15-min incubation (Figure 3c). As the incubation time increases, more $\mathrm{RNP}_{8}$ nanoparticles enter cells through endocytosis. At 1-h time point, a significant amount of internalized $\mathrm{RNP}_{8}$ (siRNA labeled with a red fluorophore) is co-localized with LysoTracker Green. This colocalization pattern diminishes after 3 hours, indicating effective endosomal escape of $\mathrm{RNP}_{8}$. Note that some red fluorescence still appears to be punctuated, likely due to newly ruptured endosomes, where siRNAs are much more concentrated than the ones that have diffused away and diluted in the cytosol. It has been shown previously by others and us that short histidine oligomers with a pKa value of 6 promote cargo cytoplasmic release due to their proton buffering capacity. ${ }^{25,}$, 29-30 At neutral $\mathrm{pH}$, they are mainly deprotonated, whereas in acidic compartments such as endosome, His protonation results in osmotic swelling and cargo release, a mechanism proposed as the proton sponge effect. ${ }^{31}$ In our current study, we inserted a short 27-amino acid peptide, $\left(\mathrm{H}_{2} \mathrm{E}\right)_{9}$, between the bulky dsRBD and branched PEG. The histidines (18 in each arm, 144 for 8 arms combined) are responsible for endosomolysis. Because His is generally hydrophobic at neutral $\mathrm{pH}, \mathrm{H}_{2} \mathrm{E}$ repeats are used to improve peptide solubility (E: glutamic acid, highly hydrophilic). The endosomolytic function of the $\mathrm{H}_{2} \mathrm{E}$ repeats is confirmed by engineering a truncated version of $\mathrm{RNP}_{8}$ where the dsRBD block is directly immobilized around the periphery of the PEG without the $\mathrm{H}_{2} \mathrm{E}$ repeats. The control $\mathrm{RNP}_{8}$ shows negligible endosomal escape after 6-h of incubation (Figure 3c).

To investigate the dissociation of $\mathrm{RNP}_{8}$ complex in cells (also known as unpackaging), we dual labeled the cargo siRNA-DUPA with a red fluorophore and the delivery vehicle, dsRBD octamer, with a green fluorophore. At early stage of $\mathrm{RNP}_{8}$ interaction with cells (15 min and $1 \mathrm{~h}$ ), the cargo and delivery vehicle remain associated as indicated by co-localization of the green and red fluorescence on cell membrane and in endosomal vesicles (merged 
color, yellow, Figure 3d). At longer incubation times ( $3 \mathrm{~h}$ and $6 \mathrm{~h}$ ), the green and red fluorescence separate. This observation provides direct evidence of active siRNA release from $\mathrm{RNP}_{8}$ into the cytoplasm that is desirable for siRNA loading into the RISC complex (a critical step in RNAi). Since dsRBD recognizes RNA in a sequence-independent fashion and binds with longer RNA with higher affinity, ${ }^{32}$ we hypothesized that the dissociation is triggered by displacement of siRNA from dsRBD by the highly abundant RNA molecules in cytosol. To verify it, $\mathrm{RNP}_{8}$ was incubated with total RNA isolated from cells. At the RNA concentration of $80 \mathrm{ng} / \mu \mathrm{L}$ (which is still much lower than the cytosolic RNA concentration), siRNA/dsRBD complex is totally disrupted (Figure 3e \& Supplementary Figure S9).

\section{In vitro gene knockdown}

To evaluate the RNAi efficacy in vitro, the DUPA-targeted $\mathrm{RNP}_{8}$ (the siRNA sequence in it is against Plk1 expression) was tested in LNCaP cells in comparison with a number of controls. To directly assess gene expression suppression, we first measured the Plk1 mRNA level in treated cells using quantitative real-time PCR. The mRNA level in cells treated with a control siRNA sequence, siRNA against Plk1, and siRNA (against Plk1)-DUPA remains unchanged compared to the untreated cells (Figure 4a). The siRNA (against Plk1)-DUPA conjugate shows excellent silencing effect, however, requiring the presence of a conventional cationic transfection agent (Lipofectamine). Similarly, the $\mathrm{RNP}_{8}$ nanoparticles exhibit a dose-dependent silencing performance. At $100 \mathrm{nM}$ siRNA (RNA 8 concentration equals to $100 / 8 \mathrm{nM}$ ), the Plk1 expression is suppressed by $60 \%$; whereas at $300 \mathrm{nM}$ siRNA, the reduction reaches approximately $74 \%$. In terms of the silencing efficacy, $\mathrm{RNP}_{8}$ is less efficient compared to Lipofectamine under the in vitro transfection condition. However, it is important to point out that Lipofectamine and other cationic carriers are not suitable for targeted in vivo delivery (except niche applications such as delivery to the liver), which is the key problem we are trying to address here. ${ }^{33-35}$ In line with the Plk1 mRNA level decreases, the Plk1 protein expression is also decreased upon $\mathrm{RNP}_{8}$ treatment (western blot analysis Figure 4b, and flow cytometry Figure 4c).

Next, we assessed the anti-proliferation effect of Plk1 silencing using the Alamar Blue assay (Figure $4 d$ ). Silencing Plk1 expression by $\mathrm{RNP}_{8}$ dramatically reduces $\mathrm{LNCaP}$ proliferation in contrast to cells treated with siRNA-DUPA. This anti-proliferation effect well correlates with cells arrested in the G2/M phase (Figure 4e). It is well-documented that cells arrested in G2/M phase may undergo apoptosis. We studied this effect by labeling the LNCaP cells with Annexin V and Propidium iodide (PI), markers for apoptosis and cell death. Our results show that in the absence of transfection reagents, $\mathrm{RNP}_{8}$ strongly induces cell apoptosis (Figure 4f). The cell death (sum of early apoptotic, late apoptotic and necrotic cell population) is $54.3 \%$ of the total cell population, in contrast to $13.2 \%$ in untreated cells and $12.5 \%$ in cells treated with siRNA-DUPA, showing the therapeutic effect of $\mathrm{RNP}_{8}$ in vitro. The presence of other apoptotic indicators such as active caspase 3 and 7 were also confirmed in cells treated with $\mathrm{RNP}_{8}$ (Supplementary Figure S12).

\section{Targeted siRNA delivery in vivo}

LNCaP and PC-3 xenografts were grown subcutaneously in immunodeficient mice. LNCaP and PC-3 cells are established prostate cancer cell lines that originated from a lymph 
node and bone metastases respectively. To probe $\mathrm{RNP}_{8}$ 's biodistribution, the DUPA-siRNA in $\mathrm{RNP}_{8}$ was labeled with Alexa Fluor 680, and its fluorescence was monitored with near infrared (NIR) imaging after intravenous injection. Three hours post-injection, mice receiving $\mathrm{RNP}_{8}$ displayed strong NIR fluorescence signals in the LNCaP tumors, but not the PC-3 tumors. More importantly, the fluorescence persisted in the LNCaP tumors for at least $24 \mathrm{~h}$ whereas fluorescence signals in other organs quickly decayed away (Figure $5 \mathrm{a} \& 5 \mathrm{~b}$, Supplementary Figure S13 \& S14). In contrast, when siRNA-DUPA was administered alone (not complexed with dsRBD octamer), a very limited quantity of siRNA-DUPA reached the tumor sites, likely due to its lower binding to tumor cells and faster clearance from systemic circulation (Figure 5c). Tumors and other major organs were dissected and imaged again for more quantitative assessment (avoid light attenuation by the skin). Consistent with the non-invasive imaging results, $\mathrm{RNP}_{8}$ accumulates in $\mathrm{LNCaP}$ tumors at a significantly higher concentration than the other organs (Figure 5a). Compared to siRNA-DUPA and $\mathrm{RNP}_{8}$ without DUPA, $\mathrm{RNP}_{8}$ carries a significant higher amount of siRNA to the tumor (Figure 5a). Confocal fluorescence imaging of $\mathrm{LNCaP}$ tumor thin sections also confirms these results. Red fluorescence from dye-labeled siRNA is easily detected in tumors treated with $\mathrm{RNP}_{8}$, but not with siRNA-DUPA alone (Figure 5d).

We then proceeded to evaluate the therapeutic activity of $\mathrm{RNP}_{8}$ in mice bearing $\mathrm{LNCaP}$ or PC-3 xenografts. $\mathrm{RNP}_{8}$ and control compounds (PBS and siRNA-DUPA) were administered systemically once every 3 days for a total of 11 doses. A pronounced inhibition of LNCaP tumor growth was observed in animals treated with $\mathrm{RNP}_{8}$. In contrast, tumors in PBS or siRNA-DUPA treated animals exhibited a sustained increase in tumor volume through the entire treatment period (Figure 6a, left panel). No body weight decrease was observed in all the treatment groups during the 1-month treatment time, showing the safety for both $\mathrm{RNP}_{8}$ and siRNA-DUPA (Figure 6b). Additional toxicity evaluations such as in vitro cytotoxicity and ex vivo histology further confirm the absence of acute toxicity (Supplementary Figure S15 and S16).

To further characterize the in vivo therapeutic effect, a number of control experiments were carried out. First, when $\mathrm{RNP}_{8}$ was given to mice bearing PC-3 xenografts (Figure 6a and 6b, right panels), no therapeutic effects (both tumor growth rate and endpoint tumor mass) were observed compared to the tumors treated with PBS, indicating the dominant effect of molecular targeting over enhanced permeability and retention (EPR). Second, $\mathrm{LNCaP}$ tumors were treated with $\mathrm{RNP}_{8}$ formulations without the DUPA targeting ligand, the cleavable disulfide bond, or the $\mathrm{H}_{2} \mathrm{E}$ repeats. As shown in Figure $6 \mathrm{c}$, virtually no anti-tumor effect is observed, demonstrating the significance of simultaneously achieving all criteria in delivery vehicle design. Third, to benchmark the $\mathrm{RNP}_{8}$ performance against existing technologies, polyethylenimine (PEI), one of best established siRNA delivery vehicles, was used to deliver siRNA-DUPA. Only minor tumor growth suppression effect was observed (Figure 6c) compared to that of $\mathrm{RNP}_{8}$. The low efficiency is likely resulted from cationic carriers' poor targeting ability and colloidal stability due to the excess positive charges. ${ }^{36-37}$

The anti-tumor effect was further examined microscopically. Histological analysis shows liquefactive necrosis-like areas in $\mathrm{LNCaP}$ tumors treated with $\mathrm{RNP}_{8}$, but not in tumors treated with PBS or siRNA-DUPA (Figure 6d, top panels). Immunohistochemistry (IHC) 
studies were also conducted to confirm Plk1 knockdown. Plk1 immunoreactivity (brown deposits) is significantly reduced in $\mathrm{RNP}_{8}$ treated $\mathrm{LNCaP}$ tumor compared to the control groups (Figure 6d, middle panels). Furthermore, using the TUNEL assay, apoptotic cells are clearly visible in $\mathrm{RNP}_{8}$-treated $\mathrm{LNCaP}$ tumors (green fluorescence in Figure 6d, bottom panels). Taken together, these results demonstrate highly specific and efficient gene silencing and anti-tumor activities of $\mathrm{RNP}_{8}$ in vivo.

\section{Preliminary evaluation of immune responses}

A remaining issue is potential immunogenicity, which is a critical factor for clinical translation. Although recombinant human proteins are not entirely free of immune-responses except of a few cases, they are generally much less immunogenic than exogenous proteins (frequency of developing immune responses to FDA-approved biologics typically limited to a few percent). ${ }^{38}$ In parallel, the chemically modification of the siRNA $\left(2^{\prime}-\mathrm{OMe}\right)$ not only improves its stability against enzymatic degradation but also helps it to escape the recruitment of toll-like receptors 7 and 8 (TLR7/8) in vivo. ${ }^{39}$ Nevertheless, we systematically probed the responses of 8 cytokines related to the innate and adaptive immunity in primary human peripheral blood mononuclear cells (PBMCs) after exposure to $\mathrm{RNP}_{8}$ or siRNA-DUPA (cargo only). Neither $\mathrm{RNP}_{8}$ nor siRNA-DUPA triggers cytokine responses (Figure 6e). In contrast, the conventional cationic PEI/siRNA complexes trigger strong responses of interleukin (IL)- $1 \beta$, tumor necrosis factor (TNF)- $\alpha$ and IL-6. To evaluate the potential immune responses in vivo, production of early innate cytokines IL- $1 \beta$, TNF- $\alpha$ and IL-6 were measured in immune competent C57/BL6 mice upon administration of $\mathrm{RNP}_{8}$ or siRNA (cargo only). Compared with the negative control (PBS buffer), neither agent alters cytokine production above background levels, unlike LPS, the positive control (Figure 6f).

\section{DISCUSSIONS}

siRNA is one of the most valuable tools for investigation of gene function and holds great potential for use in clinical settings. However, the obstacle preventing its clinical translation is targeted delivery in vivo, a chronic problem associated with virtually all antisense therapies. Current nanocarriers including liposomes, polymers, peptides, and inorganic nanoparticles all suffer from one or another limitation (such as large particle size, aggregation in serum, cytotoxicity, and immune response), which helps explain the setbacks in clinical translation and commercialization. In particular, the need of cationic carrier materials for oligonucleotide condensation, protection and endosomal escape, and the need of neutral or negative surface charges for specific targeting are seemingly two conflicting design requirements, preventing the formulation of an ideal delivery system.

In this report, we describe the development of a universal siRNA nanocarrier that simultaneously achieves all the desired features such as RNA binding, endosomal escape, compact size, high payload, and biosafety. The various domains of our multifunctional designer nanocarrier are predominately composed of a small protein in the human proteome repertoire and PEG, arguably the most versatile biocompatible polymer discovered to date. The fully assembled nanocarrier features a discrete number of binding sites, which are not 
available in conventional liposomes, cationic polymers, and inorganic nanoparticles, and can stably immobilize virtually any double-stranded RNA molecule. Extensive biochemical experiments show that 16 bp of dsRNA binds to dsRBD at nM affinity. ${ }^{12,14}$ This flexibility in selection of siRNA as well as their conjugates with various types of targeting ligands make this class of multifunctional nanocarrier an ideal system for targeted siRNA delivery (Supplementary Figure S17). Indeed, we and others have previously genetically modified dsRBD for siRNA binding. ${ }^{12,}$ 25, 40-42 These modifications, however, are either missing critical functionalities, ${ }^{41}$ immunogenic, ${ }^{25}$ or converting dsRBD back to the conventional cationic carriers. ${ }^{12,40,42}$ In our design, the dsRBD not only provides RNA binding functionality, but also serves as a bulky protein shield, hiding short non-natural peptides from the immune surveillance.

The dsRBD octamer offers a remarkably high payload for siRNA ( $38 \%$ by weight), while still maintains a compact size and excellent colloidal stability in buffers and serum (Supplementary Figure S18). Both TEM and DLS measurements indicate that the fully assembled $\mathrm{RNP}_{8}$ complex is approximately $30 \mathrm{~nm}$ in diameter, which is in excellent size range for in vivo targeting and drug delivery. ${ }^{26}$ The compact and uniform size is a result of molecular self-assembly, in contrast to electrostatic aggregation (often forms large and heterogeneous particles).

In addition to RNA loading, endosomal escape is another critical factor for RNAi. Endosomal disruption by a $\mathrm{pH}$-responsive histidine-rich peptide is an effective way to achieve this goal. ${ }^{25}, 29-30$ Unlike the dsRBD of human origin and the PEG that has been widely used in pharmaceutics, the short $\left(\mathrm{H}_{2} \mathrm{E}\right)_{9}$ peptide is not a natural sequence in the human proteome. In our in vivo experiments, the peptide is not of concern since mouse xerograph models are immune compromised for tumor growth. In patients, it might trigger minor immune responses, despite the fact that short peptides typically have lower immunogenicity than large proteins, and hundreds of recombinant proteins have received approval worldwide. ${ }^{43}$ To help reduce the potential immunogenicity and minimize risk for future clinical trials, we chose 8-armed PEG, a spatially crowded molecule, as the assembly scaffold. In this design, the short $\left(\mathrm{H}_{2} \mathrm{E}\right)_{9}$ peptide is sandwiched between two bulky molecules, the dsRBD of $\sim 20 \mathrm{kDa}$, and the branched PEG, sterically hindering it from interacting with biomacromolecules such as antibodies, yet still remaining accessible to small ions (buffering $\mathrm{H}^{+}$to help destabilize endosome). It was recently shown that a brush PEG structure (also spatially crowded) not only improves peptide drug pharmacokinetics but also dramatically reduces immunogenicity. ${ }^{44}$ Indeed, when $\mathrm{RNP}_{8}$ was evaluated for cytokine responses in PBMCs and mice, no over-expression was detected, in contrast to PEI-based delivery.

In this study, targeting is achieved with a small molecule-ligand linked to the siRNA through a cleavable bond. Taking advantage of the sequence independent binding of dsRBD, the siRNA end of the siRNA-DUPA conjugate docks into dsRBD, rendering the targeting ligand accessible. Although the conventional cationic delivery vehicles are extremely efficient in cell transfection, they generally have poor in vivo biodistribution profiles owing to nonspecific electrostatic interactions with the biological systems. ${ }^{33-35}$ The $\mathrm{RNP}_{8}$ avoids this issue utilizing a natural protein, dsRBD. With numerous siRNA conjugates linking siRNAs 
with targeting ligands ranging from macromolecular antibody to peptides, aptamers, and small molecules, ${ }^{11,16,45-46}$ personalized treatment can become available and affordable. In our current study, the DUPA ligand and siRNA are linked via a cleavable chemical bond, and the impact of this design feature on RNAi is significant. The disulfide bond is relatively stable in circulation and the extracellular space, but prone to rapid cleavage in the reductive intracellular space (due to higher concentration of glutathione).$^{47}$ The resulting siRNA without the bulky targeting ligand allows siRNA-RISC interaction without steric hindrance, thus is more potent in RNAi. ${ }^{48}$ Similar results have been previously observed for other siRNA conjugates such as with cholesterol and PEI. ${ }^{47,49}$

To evaluate the performance of the $\mathrm{RNP}_{8}$, we have assessed targeted siRNA delivery both in vitro and in vivo. Cell-specific gene knockdown was observed under both conditions, due to multivalent specific targeting, compact particle size, and absence of positive surface charge. Under in vitro condition, Lipofectamine still outperforms $\mathrm{RNP}_{8}$ in gene silencing. This is expected, as cationic transfection agents are proven to be effective in DNA and RNA transfection (binding with cell surface via charge-charge interactions is much faster than molecular recognition). But, they are not suitable for systemic targeted (non-liver) delivery in vivo, which has been a long-standing problem for many biologics. ${ }^{33-35}$ Notably, under in vivo conditions, the DUPA-targeted $\mathrm{RNP}_{8}$ substantially suppressed the growth of PSMA-positive LNCaP, but showed virtually no-effect in PSMA-negative PC-3 xenografts, indicating excellent targeting specificity. At the same time, the implanted tumors were not completely eliminated, as tumor cells can survive through alternative signaling pathways that promote proliferation and inhibit apoptosis. Theoretically, RNAi can control the activity of all these pathways. We envision that potentially personalized cocktail therapies by combining different siRNA sequences, siRNA and chemotherapy, and siRNA and radiotherapy, will be much more effective in treating cancer. These exciting opportunities will be explored in the future after development and optimization of the in vivo delivery technology.

In conclusion, the key obstacle preventing the powerful siRNA technology from clinical translation is targeted delivery in vivo, a chronic problem plaguing virtually all antisense therapies, ${ }^{50}$ and potentially a major problem for in vivo uses of the genome editing technology in the future. ${ }^{3-6}$ On the basis of the concepts of engineering and assembling natural proteins, and of sterically hiding short non-natural peptides between two bulky macromolecules for additional functionalities, we have developed an efficient siRNA delivery system that achieves all the desired design properties. Most importantly, RNA loading and endosomal escape are simultaneously achieved without excessive cationic surface changes. This delivery technology also features multivalency, enabling efficient cell uptake and potentially allowing combination of virtually any RNA sequences for combinatorial therapy. We believe that further development and clinical testing of the $\mathrm{RNP}_{8}$ technology will raise exciting opportunities for biologics therapy. 


\section{METHODS}

\section{Cell culture}

LNCaP, 22RV1 and PC-3 cells were purchased from American Type Culture Collection (ATCC, Manassas, VA). LNCaP and 22RV1 cells were cultured in RMPI 1640 (LonzaBioWhittaker, Allendale, NJ) supplemented with $25 \mathrm{mM}$ HEPES, 10\% FBS and antibiotics (penicillin: 100 units/mL, streptomycin: $100 \mu \mathrm{g} / \mathrm{mL}$ ). PC-3 cells were cultured in DMEM (Corning, Corning, NY) supplemented with 10\% FBS and antibiotics (penicillin: 100 units $/ \mathrm{mL}$, streptomycin: $100 \mu \mathrm{g} / \mathrm{mL}$ ). All cells were incubated at $37^{\circ} \mathrm{C}$ with $5 \% \mathrm{CO}_{2}$.

\section{dsRBD expression, octamer formation, and purification}

The protein expression vector was constructed by separately cloning the cDNA of the dsRBD domain of human PKR (cDNA of aa 1-172), cDNA of the $\left(\mathrm{H}_{2} \mathrm{E}\right)_{9}$ peptide and Sortase A substrate peptide (cDNA synthesized by IDT, Coralville, IW) ) into the pET30a vector (the protein sequence of the whole construct is available in Supplementary Figure $\mathrm{S} 1)$. The $\left(\mathrm{H}_{2} \mathrm{E}\right)_{9}$ peptide domain can be used for protein purification because it is histidine rich. The dsRBD protein was expressed in a codon-plus BL21 strain, Rossetta 2(DE3), under auto-induction condition at RT for $36 \mathrm{~h}^{51}$. The cells were recovered by centrifugation $(5,000 \mathrm{~g} \times 5 \mathrm{~min})$ and lysed by the B-PER ${ }^{\mathrm{TM}}$ extraction reagent (Thermofisher, Waltham, MA). The soluble proteins were isolated by centrifugation for $15 \mathrm{~min}$ at $9,000 \mathrm{~g}$ and dsRBD was purified from the supernatant by Ni-affinity chromatography, followed by another round of purification using ion-exchange chromatography (Hiscreen ${ }^{\mathrm{TM}} \mathrm{Q}$ column, $\mathrm{pH}$ 8.5). The purified dsRBD protein was diluted to $2 \mathrm{mg} / \mathrm{mL}$ in TBS buffer and ligated to $\mathrm{NH}_{2}$-GG$\mathrm{PEG}_{3}-\mathrm{N}_{3}(10 \mathrm{mM})$ in the presence of Sortase A $(0.25 \mu \mathrm{M})$ (overnight at RT). The azidefunctionalized dsRBD was precipitated with ammonium sulfate and further purified with ion-exchange chromatography to remove free $\mathrm{NH}_{2}-\mathrm{GG}-\mathrm{PEG}_{3}-\mathrm{N}_{3}$. To graft dsRBD-azide onto the branched PEG, $5 \mathrm{mg} / \mathrm{ml}$ in TBS buffer containing $20 \%$ glycerol was incubated with 8-arm PEG-DBCO (m.w. 20k, 0.0125 mM, JenKemUSA Inc, Plano, TX) at RT overnight. The dsRBD octamer was purified by gel filtration chromatography (Superdex 200 HR 10/30 column, GE lifesciences, Pittsburgh, PA) using TBS as the elution buffer. The purified octamer was concentrated and stored in TBS buffer containing $20 \%$ glycerol at $-30{ }^{\circ} \mathrm{C}$.

\section{SiRNA conjugation}

All the reagents and organic solvent were purchased from Sigma-Aldrich. DUPA-PEG ${ }_{11^{-}}$ $\mathrm{NH}_{2}$ was synthesized according to previous reports ${ }^{52}$. DBCO was coupled to the amino group of DUPA-PEG $11-\mathrm{NH}_{2}$ by incubating the DUPA ligand with DBCO-NHS in anhydrous dichloromethane overnight in the presence of an acid scavenger, triethylamine. The product was then precipitated by adding ethyl ether. The crude compound (DUPA-PEG ${ }_{11}-\mathrm{DBCO}$ ) was dissolved in $20 \mathrm{mM}$ Tris-Cl buffer (pH 8.5) and purified by HiTrap ${ }^{\circledR}$ Q HP column. The molecular weight was confirmed by mass spectrometry (Supplementary Figure S2).

siRNAsequences were purchased from Integrated DNA Technologies, Inc. (IDT, Inc. Coralville, IW). The siRNA sequences were stabilized by using $2^{\prime}$-O-methylated bases (underline), $2^{\prime}$-fluoro bases (italics), or phosphorothioate bond substitution (*). The sequences are: Plk1 sense strand (unmodified): AGAAGAUGCUUCAGACAGATT; 
Plk1 antisense strand (unmodified): UCUGUCUGAAGCAUCUUCUTT; Plk1 sense strand $\left(2^{\prime}\right.$-OMe): $/ 5^{\prime}$ Thiol- $\mathrm{C}_{6} / \mathrm{A} \underline{\mathrm{G}} \underline{\mathrm{A}} \underline{\mathrm{G}} \underline{\mathrm{A}} \underline{\mathrm{G}} \underline{\mathrm{U}} \underline{\mathrm{U}} \underline{\mathrm{A}} \mathrm{G} A \underline{\mathrm{C}} \mathrm{A} \underline{\mathrm{G}} \mathrm{A} * \mathrm{~T} * \mathrm{~T} ; \mathrm{Plk} 1$

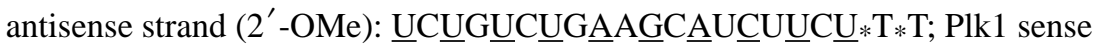
strand $\left(2^{\prime}-\mathrm{F}\right): / 5^{\prime}$ Thiol- $\mathrm{C}_{6} /$ AGAAGA $U$ G $C U U C A G A C A G A * \mathrm{~T} * \mathrm{~T}$; Plk1 antisense strand (2'-F): UCUGUCUGAAGCAUCUUCU*T $* \mathrm{~T}$; Control sense strand $\left(2^{\prime}\right.$ -

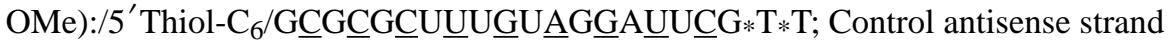

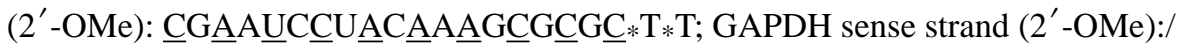
$5^{\prime}$ Thiol- $\mathrm{C}_{6} / \mathrm{C} \underline{\mathrm{A}} \underline{\mathrm{C}} \underline{\mathrm{U}} \underline{\mathrm{U}} \underline{\mathrm{C}} \underline{\mathrm{C}} \underline{\mathrm{U}} \underline{\mathrm{C}} \underline{\mathrm{U}} \underline{\mathrm{U}} \underline{\mathrm{U}} \mathrm{A} \underline{\mathrm{C}} \mathrm{U} * \mathrm{~T} * \mathrm{~T}$; Plk1 antisense strand $\left(2^{\prime}-\mathrm{OMe}\right)$ : $\underline{A} \underline{G} \underline{U} \underline{G} A \underline{G G} \underline{C} A \underline{G G} \underline{G} A \underline{U} G \underline{A} \underline{G} * T * T ;$ GAPDH antisense strand (unmodified): UCUGUCUGAAGCAUCUUCUTT. To add an azide group, the sense strands with a thiol group (pre-reduced with $2 \mathrm{mM}$ TCEP solution and precipitated with alcohol) was incubated with excess amount of $\mathrm{N}_{3}-\mathrm{PEG}_{3}$-OPSS $(20 \mathrm{mM})$ overnight at RT. The crude RNA was precipitated with alcohol and purified by HiTrap ${ }^{\circledR}$ Q HP column.

The DUPA ligand was coupled to RNA by incubating DUPA-PEG $11-\mathrm{DBCO}_{1}$ with the azidemodified sense strand via copper-free click reaction. The resulting construct was purified and hybridized with the siRNA antisense strand to create the siRNA-DUPA conjugate. The siRNA conjugate was resolved with 5\% agarose gel and visualized with Gel-Red staining to confirm its size and purity.

\section{$\mathrm{RNP}_{\mathbf{8}}$ self-assembly and stability}

The $\mathrm{RNP}_{8}$ complex was obtained by incubating the dsRBD octamer and siRNA at a molar ratio of 1:8 at RT for $5 \mathrm{~min}$. The assembly was tested for stability or used for silencing experiments without further purification. For stability test, the $\mathrm{RNP}_{8}$ complex was incubated in $50 \%$ mouse serum at $37{ }^{\circ} \mathrm{C}$. The siRNA in the complex was labeled with FAM (Thermofisher, Waltham, MA). Aliquots of $5 \mu \mathrm{L}$ (10 pmol siRNA equivalent) was collected at various time points and probed on $1 \%$ agarose gel. The gel images were obtained using a fluorescence imaging system (Light Tools Research, Pasadena, CA) and a Typhoon 7000 scanner (GE healthcare, UK). The cargo payload was calculated by dividing the total weight of 8 copies of siRNA ( $112 \mathrm{KDa})$ by the total weight of the entire RNP8 ( 292KDa) which contains 8 copies of siRNA, 8 copies of dsRBD, and an 8 -arm PEG.

\section{TEM characterization}

Formvar-coated TEM grids (Electron Microscopy Sciences, Hatfield, PA) were incubated with $10 \mathrm{uL}$ of protein solution for $2 \mathrm{~min}$, washed twice with D.D. water and negatively stained with $0.75 \%$ Uranyl formate for 30 seconds. The grids were air-dried and stored at R.T. in dark until image acquisition. Images were obtained on a Tecnai TF20 microscope (FEI Corporate, Hillsboro, OR).

\section{Cellular uptake, flow cytometry and confocal microscopy}

To evaluate the cell binding ability of $\mathrm{RNP}_{8}$, it was incubated with LNCaP, 22RV1 and PC-3 cells at RT to minimize internalization. Briefly, $\mathrm{RNP}_{8}$ (the siRNA in it was fluorescently labeled with Alexa Fluor 555) and cells ( 40,000) were incubated for $1 \mathrm{~h}$ in RPMI 1640 supplemented with $1 \%$ BSA. The cells were washed with PBS and their fluorescence was measured on a LSR II flow cytometer (Beckton Dickinson, Franklin Lakes, NJ). A 
representative gating strategy for the flow cytometry analysis is shown in Supplementary Fig. 19. For cellular uptake, $\mathrm{RNP}_{8}$ incubation with cells was performed at $37^{\circ} \mathrm{C}$. At the end of the incubation, cells were lysed with PBS containing $0.25 \%$ Triton X-100, and the uptake was measured by quantifying the siRNA in $\mathrm{RNP}_{8}$ in cell lysate using stem-loop real-time $\mathrm{PCR}^{53}$. To image $\mathrm{RNP}_{8}$ intracellular trafficking, dual-color fluorescence-labeled $\mathrm{RNP}_{8}$ (siRNA labeled with Alexa Fluor 555 and the dsRBD octamer labeled with Alexa Fluor 488) was incubated with LNCaP cells at $37^{\circ} \mathrm{C}$ and imaged at $0 \mathrm{~min}, 15 \mathrm{~min}, 1 \mathrm{~h}, 3 \mathrm{~h}$ and $6 \mathrm{~h}$. In a separate experiment, single-color fluorescence-labeled $\mathrm{RNP}_{8}$ (only the siRNA is labeled with Alexa Fluor 555) was incubated with cells together with LysoTracker Green DND-26 (Thermofisher, Waltham, MA). After counter-stained with Hoechst, the cells were analyzed with a LSM 510 Meta confocal microscpe (Zeiss, Dublin, CA).

\section{SPR assay}

A Biacore T200 instrument was used for this assay (Biacore, Piscataway, NJ). PSMA (R\&D system, Inc, Minneapolis, MN) was diluted to $10 \mu \mathrm{g} / \mathrm{ml}$ in acetic acid buffer (pH 5.0) and immobilized onto CM5 sensor chips (at $\approx 9,000$ resonance units for the siRNA-DUPA test and at $\approx 1,000$ resonance units for the $\mathrm{RNP}_{8}$ test) at a flow rate of $10 \mu \mathrm{l} / \mathrm{min}$ according to the manufacturer's instructions. A reference surface was used as the background control. Binding characteristics was evaluated at $25^{\circ} \mathrm{C}$ in DPBS buffer with $0.005 \%$ (vol $/ \mathrm{vol}$ ) surfactant, P20. SiRNA-DUPA of various concentrations $(0 \mathrm{nM}, 25 \mathrm{nM}, 50 \mathrm{nM}, 75 \mathrm{nM}$ and $100 \mathrm{nM})$ and $\mathrm{RNP}_{8}(0 \mathrm{nM}, 5 \mathrm{nM}, 10 \mathrm{nM}, 15 \mathrm{nM}$ and $20 \mathrm{nM})$ were passed through the control and test flow cells at a flow rate of $30 \mu \mathrm{l} / \mathrm{min}$. The test was run in a single cycle kinetic model without regeneration of the surface.

\section{In vitro RNAi and quantitative measurements}

$\mathrm{RNP}_{8}$ was assembled by incubating the dsRBD octamer and siRNA-DUPA in DPBS for 5 min under sterile condition. $2 \times 10^{5} \mathrm{LNCaP}$ cells were treated with fresh $\mathrm{RNP}_{8}$ for $12 \mathrm{~h}$ in RPMI 1640 media plus $10 \%$ Q-serum ${ }^{42,54}$, followed by a media exchange. As a positive control, cells were treated with $100 \mathrm{nM}$ siRNA in the presence of Lipofectamine 2000 according to the manufacturer's recommended protocol (Thermofisher, Waltham, MA). Thirty-two hours post-treatment, cells were harvested for RT-PCR and immunoblotting. Briefly, total RNA was isolated and reverse transcribed into cDNA using random hexamer and Taqman ${ }^{\circledR}$ Reverse Transcription reagents (Thermofisher, Waltham, MA). mRNA expression was measured using SensiFast SYBR kit (Bioline, Taunton, MA) on Chromo4 ${ }^{\mathrm{TM}}$ Real-Time system (Bio-Rad, Hercules, CA). Plk1 mRNA expression level was normalized to peptidylprolyl isomerase A (PPIA) and expressed as the percentage of negative control. Immunoblotting was performed as previously reported ${ }^{55}$ and all antibodies were purchased from ThermoFisher. The immunoblots were imaged on an Odyssey ${ }^{\circledR} \mathrm{CLx}$ imaging system (Li-cor, Lincoln, NE) and analyzed with Image Studio Lite.

\section{Cell viability, cell cycle and apoptosis assays}

LNCaP cells were treated with $300 \mathrm{nM}$ of siRNA-DUPA or $\mathrm{RNP}_{8}$. Un-treated cells were used as the negative control, whereas siRNA-DUPA with transfection agents was used as the positive control. The cells were processed on the third day after treatment. Cell viability was measured using the CellTiter-Blue ${ }^{\circledR}$ (AlamarBlue) Cell Viability Assay kit (Promega, 
Madison, WI), following the manufacturer's protocol. To probe the cell cycle profiles, cells were first trypsinized with TrypLETM Express Enzyme reagent (Thermofisher, Waltham, MA) and washed twice with DPBS. After incubation with Vybrant ${ }^{\circledR}$ DyeCycle ${ }^{\mathrm{TM}}$ Orange Stain (Thermofisher, Waltham, MA) at $37^{\circ} \mathrm{C}$ for $30 \mathrm{~min}$, the stained cells were analyzed on a flow cytometer using $532 \mathrm{~nm}$ excitation. To test apoptosis, cells were stained with Alexa Fluor 488-labeled annexin V and propidium iodide (Thermofisher, Waltham, MA) for 15 min at RT. Cell fluorescence was measured with flow cytometry.

\section{In vivo biodistribution and targeted RNAi}

All protocols were approved by the University of Washington, International Animal Care and Use Committee (IACUC). LNCaP or PC-3 cells were trypsinized, washed with PBS, and resuspended in Matrigel ${ }^{\mathrm{TM}}$ (BD Biosciences, San Jose, CA). $5 \times 10^{6}$ cells were injected subcutaneously into 6-8 week-old male $\mathrm{Nu} / \mathrm{Nu}$ mice (Charles River, Wilmington, MA). Tumor growth was measured twice a week and the size was calculated using formula length $\times$ width $^{2} / 2$. For in vivo biodistribution studies, $\mathrm{RNP}_{8}$ with Alexa Fluor 680 -labeled siRNADUPA ( $1 \mathrm{mg} / \mathrm{kg}$, total volume of $250 \mu \mathrm{L})$ was administrated into mice bearing LNCaP tumors $\left(400-600 \mathrm{~mm}^{3}\right)$ via tail vein injection. Near-infrared (NIFR) optical images were taken on an IVIS imaging system (Perkinelmer, Waltham, MA) 3, 8 and 24 h post injection. At the endpoint ( $3 \mathrm{~h}, 8 \mathrm{~h}$ and $24 \mathrm{~h}$ post injection), mice were euthanized and the tumors as well as other major organs were harvested for ex vivo imaging. The fluorescence intensities of tissues were analyzed with the Living Image Software from Perkinelmer (Waltham, MA) and normalized to the weight of the corresponding tissues.

For s.c. tumor challenge, mice were intravenously injected with PBS, of siRNA-DUPA alone (4.3 nmol), $\mathrm{RNP}_{8}$ (equivalent amount of siRNA) or $\mathrm{RNP}_{8}$ missing certain functionalities (DUPA ligand, cleavable disulfide linkage, or the $\mathrm{H}_{2} \mathrm{E}$ repeats) after the tumor sizes have reached $100 \mathrm{~mm}^{3}$. The PEI/siRNA-DUPA polyplex was prepared according the literature ${ }^{56}$ and intravenously administered to tumor-bearing mice as a control. Tumor growth was monitored three times a week. Intravenous injections were repeated every three day for 32 days (LNCaP) and 25 days (PC-3). The body weight and tumor mass were also measured at the endpoint of the treatment. The growth curves were plotted as mean tumor volume \pm SEM.

\section{Histochemical analysis}

Tissues were fixed in $4 \%$ paraformaldehyde/PBS for $6 \mathrm{~h}$ and placed into a $30 \%$ sucrose/PBS solution overnight at $4{ }^{\circ} \mathrm{C}$. The fixed tissues were cut into $6 \mu \mathrm{m}$-thick sections with a cryostat. The sections were stained with hematoxylin and eosin (H\&E) for histopathological assay, or processed with TUNEL using the In Situ Cell Death Detection Kit (Roche, Pleasanton, CA). For immunostaining of Plk1, slides were blocked with $3 \% \mathrm{H}_{2} \mathrm{O}_{2}$-methanol for $15 \mathrm{~min}$ at room temperature, treated with mouse anti-Plk1 antibody (Thermofisher, Waltham, MA) overnight at $4^{\circ} \mathrm{C}$, with HRP-conjugated secondary antibody, and with DAB. The tissue sections were counterstained with hematoxylin for nucleus visualization. To image siRNA penetration into solid tumors, mice were given $\mathrm{RNP}_{8}$ containing Alexa Fluor 555-labeled DUAP-siRNA. Tumors were harvest $4 \mathrm{~h}$ post injection, fixed, and cryosectioned. The specimens were counterstained with Alexa Fluor 488 phalloidin (10 
$\mathrm{nM}$, Thermofisher, Waltham, MA) and mounted in Pro-long diamond mounting media (Thermofisher, Waltham, MA). The slides were analyzed on a LSM 510 Meta confocal microscope.

\section{Evaluation of potential immune responses}

Human peripheral blood mononuclear cells (PBMC, Zen-bio, Research Triangle Park, NC) were washed twice in RPMI 1640 and resuspended in complete culture media at a concentration of $5 \times 10^{6}$ cells $/ \mathrm{mL}$. $100 \mu \mathrm{L}$ of cell suspension was seeded into 96-well plate $\left(5 \times 10^{5}\right.$ cells/well) and treated with PBS, $200 \mathrm{nM}$ siRNA, RNP 8 , PEI/siRNA complex, or LPS $(10 \mu \mathrm{g} / \mathrm{mL})$ and PHA $(20 \mu \mathrm{g} / \mathrm{mL})$. The cell culture supernatants were collected after $24 \mathrm{~h}$ and $72 \mathrm{~h}$ incubation. Cytokines were analyzed using LEGENDplex ${ }^{\mathrm{TM}}$ Human Cytokine immunoassay (Biolegend, San Diego, CA).

To evaluate potential in vivo immune responses, immune competent C57BL6 mice were injected with PBS, siRNA ( $2 \mathrm{mg} / \mathrm{kg}$ ), $\mathrm{RNP}_{8}$ or LPS ( $2 \mu \mathrm{g}$ per mouse) intravenously. Two hours post administration, mice were sacrificed and the blood was collected for cytokine analysis using LEGENDplex ${ }^{\mathrm{TM}}$ mouse Cytokine immunoassay kit (Biolegend, San Diego, CA).

\section{Life Sciences Reporting Summary}

Further information on experimental design is available in the Life Sciences Reporting Summary.

\section{Data Availability}

The authors declare that all data supporting the findings of this study are available within the paper and its Supplementary Information.

\section{Supplementary Material}

Refer to Web version on PubMed Central for supplementary material.

\section{Acknowledgments}

This work was supported in part by NIH (R01CA140295), the Department of Bioengineering, and the Office of Research at University of Washington. We are also grateful to Drs. Lige Tonggu and Liguo Wang and for help with TEM measurements and data interpretation; Dr. Bob Vessella for help with tumor model establishment, and Dr. John Sumida at the UW Analytical Biopharmacy core for help with the SPR measurements.

\section{References}

1. Hannon GJ. RNA interference. Nature. 418: 244-251. 2002. [PubMed: 12110901]

2. Scherer LJ, Rossi JJ. Approaches for the sequence-specific knockdown of mRNA. Nat Biotechnol. 21: 1457-1465. 2003. [PubMed: 14647331]

3. Bahal R, et al. In vivo correction of anaemia in beta-thalassemic mice by gammaPNA-mediated gene editing with nanoparticle delivery. Nat Commun. 7: 13304. 2016; [PubMed: 27782131]

4. Cox DB, Platt RJ, Zhang F. Therapeutic genome editing: prospects and challenges. Nat Med. 21: 121-131. 2015. [PubMed: 25654603]

5. Nelson CE, et al. In vivo genome editing improves muscle function in a mouse model of Duchenne muscular dystrophy. Science. 351: 403-407. 2015. [PubMed: 26721684] 
6. Suzuki K, et al. In vivo genome editing via CRISPR/Cas9 mediated homology-independent targeted integration. Nature. 540: 144-149. 2016. [PubMed: 27851729]

7. Ashley CE, et al. The targeted delivery of multicomponent cargos to cancer cells by nanoporous particle-supported lipid bilayers (vol 10, pg 389, 2011). Nat Mater. 10: 476-476. 2011.

8. Kumar P, et al. Transvascular delivery of small interfering RNA to the central nervous system. Nature. 448: 39-43. 2007. [PubMed: 17572664]

9. Whitehead KA, Langer R, Anderson DG. Knocking down barriers: advances in siRNA delivery. Nat Rev Drug Discov. 8: 129-138. 2009. [PubMed: 19180106]

10. Pecot CV, Calin GA, Coleman RL, Lopez-Berestein G, Sood AK. RNA interference in the clinic: challenges and future directions. Nat Rev Cancer. 11: 59-67. 2011. [PubMed: 21160526]

11. Nair JK, et al. Multivalent N-acetylgalactosamine-conjugated siRNA localizes in hepatocytes and elicits robust RNAi-mediated gene silencing. J Am Chem Soc. 136: 16958-16961. 2014. [PubMed: 25434769]

12. Eguchi A, et al. Efficient siRNA delivery into primary cells by a peptide transduction domaindsRNA binding domain fusion protein. Nat Biotechnol. 27: 567-571. 2009. [PubMed: 19448630]

13. Kanasty R, Dorkin JR, Vegas A, Anderson D. Delivery materials for siRNA therapeutics. Nat Mater. 12: 967-977. 2013. [PubMed: 24150415]

14. Bevilacqua PC, Cech TR. Minor-groove recognition of double-stranded RNA by the doublestranded RNA-binding domain from the RNA-activated protein kinase PKR. Biochemistry. 35: 9983-9994. 1996. [PubMed: 8756460]

15. Bagci H, Kohen F, Kuscuoglu U, Bayer EA, Wilchek M. Monoclonal anti-biotin antibodies simulate avidin $m$ the recognition of biotin. FEBS Lett. 322: 47-50. 1993. [PubMed: 8482366]

16. Dassie JP, et al. Systemic administration of optimized aptamer-siRNA chimeras promotes regression of PSMA-expressing tumors. Nat Biotechnol. 27: 839-846. 2009. [PubMed: 19701187]

17. McNamara JO, et al. Cell type-specific delivery of siRNAs with aptamer-siRNA chimeras. Nat Biotechnol. 24: 1005-1015. 2006. [PubMed: 16823371]

18. Thiel KW, et al. Delivery of chemo-sensitizing siRNAs to HER2+-breast cancer cells using RNA aptamers. Nucleic Acids Res. 40: 6319-6337. 2012. [PubMed: 22467215]

19. Neff CP, et al. An Aptamer-siRNA Chimera Suppresses HIV-1 Viral Loads and Protects from Helper CD4(+) T Cell Decline in Humanized Mice. Sci Transl Med. 3: 66ra66-66ra66. 2011.

20. Kularatne SA, Wang K, Santhapuram HKR, Low PS. Prostate-Specific Membrane Antigen Targeted Imaging and Therapy of Prostate Cancer Using a PSMA Inhibitor as a Homing Ligand. Mol Pharm. 6: 780-789. 2009. [PubMed: 19361233]

21. Chang SS, Reuter VE, Heston WDW, Gaudin PB. Metastatic renal cell carcinoma neovasculature expresses prostate-specific membrane antigen. Urology. 57: 801-805. 2001. [PubMed: 11306418]

22. Bander NH, et al. Targeting metastatic prostate cancer with radiolabeled monoclonal antibody J591 to the extracellular domain of prostate specific membrane antigen. J Urol. 170: 1717-1721. 2003. [PubMed: 14532761]

23. Nanduri S, Carpick BW, Yang YW, Williams BRG, Qin J. Structure of the double-stranded RNAbinding domain of the protein kinase PKR reveals the molecular basis of its dsRNA-mediated activation. Embo J. 17: 5458-5465. 1998. [PubMed: 9736623]

24. Ryter JM, Schultz SC. Molecular basis of double-stranded RNA-protein interactions: structure of a dsRNA-binding domain complexed with dsRNA. EMBO J. 17: 7505-7513. 1998. [PubMed: 9857205]

25. Liu HY, Gao X. A universal protein tag for delivery of SiRNA-aptamer chimeras. Sci Rep. 3: 3129. 2013; [PubMed: 24196104]

26. Cabral H, et al. Accumulation of sub-100 nm polymeric micelles in poorly permeable tumours depends on size. Nat Nanotechnol. 6: 815-823. 2011. [PubMed: 22020122]

27. Krishnamurthy, VM, Estroff, LA, Whitesides, GM. Fragment-based Approaches in Drug Discovery. Wiley-VCH Verlag GmbH \& Co. KGaA; 2006. 11-53.

28. Badjic JD, Nelson A, Cantrill SJ, Turnbull WB, Stoddart JF. Multivalency and Cooperativity in Supramolecular Chemistry. Acc Chem Res. 38: 723-732. 2005. [PubMed: 16171315] 
29. Midoux P, Pichon C, Yaouanc JJ, Jaffrès PA. Chemical vectors for gene delivery: a current review on polymers, peptides and lipids containing histidine or imidazole as nucleic acids carriers. $\mathrm{Br} \mathrm{J}$ Pharmacol. 157: 166-178. 2009. [PubMed: 19459843]

30. Pichon C, Goncalves C, Midoux P. Histidine-rich peptides and polymers for nucleic acids delivery. Adv Drug Deliv Rev. 53: 75-94. 2001. [PubMed: 11733118]

31. Behr JP. The proton sponge: A trick to enter cells the viruses did not exploit. Chimia. 51: 34-36. 1997.

32. Schmedt C, et al. Functional-Characterization of the Rna-Binding Domain and Motif of the Double-Stranded Rna-Dependent Protein-Kinase Dai (Pkr). J Molr Biol. 249: 29-44. 1995.

33. Buyens K, De Smedt S, Demeester J, Sanders N. Why simple cationic liposome formulations fail to deliver siRNA efficiently in vivo. Hum Gene Ther. 18: 1070-1070. 2007.

34. Merkel OM, et al. Stability of siRNA polyplexes from poly(ethylenimine) and poly(ethylenimine)g-poly(ethylene glycol) under in vivo conditions: Effects on pharmacokinetics and biodistribution measured by Fluorescence Fluctuation Spectroscopy and Single Photon Emission Computed Tomography (SPECT) imaging. J Control Release. 138: 148-159. 2009. [PubMed: 19463870]

35. Wu SY, Lopez-Berestein G, Calin GA, Sood AK. RNAi Therapies: Drugging the Undruggable. Sci Transl Med. 6: 240ps7. 2014;

36. Gao S, et al. The effect of chemical modification and nanoparticle formulation on stability and biodistribution of siRNA in mice. Mol Ther. 17: 1225-1233. 2009. [PubMed: 19401674]

37. Lee SY, et al. Stability and cellular uptake of polymerized siRNA (poly-siRNA)/polyethylenimine (PEI) complexes for efficient gene silencing. J Control Release. 141: 339-346. 2010. [PubMed: 19836427]

38. Parenky A, et al. New FDA draft guidance on immunogenicity. AAPS J. 16: 499-503. 2014. [PubMed: 24682766]

39. Hamm S, et al. Alternating $2^{\prime}$-O-ribose methylation is a universal approach for generating nonstimulatory siRNA by acting as TLR7 antagonist. Immunobiology. 215: 559-569. 2010. [PubMed: 19854535]

40. Li H, Zheng X, Koren V, Vashist YK, Tsui TY. Highly efficient delivery of siRNA to a heart transplant model by a novel cell penetrating peptide-dsRNA binding domain. Int J Pharm. 469: 206-213. 2014. [PubMed: 24768403]

41. Haroon MM, et al. A designed recombinant fusion protein for targeted delivery of siRNA to the mouse brain. J Control Release. 228: 120-131. 2016. [PubMed: 26948382]

42. Geoghegan JC, Gilmore BL, Davidson BL. Gene Silencing Mediated by siRNA-binding Fusion Proteins Is Attenuated by Double-stranded RNA-binding Domain Structure. Mol Ther Nucleic Acids. 1: e53. 2012; [PubMed: 23629028]

43. Sanchez-Garcia L, et al. Recombinant pharmaceuticals from microbial cells: a 2015 update. Microb Cell Fact. 15: 33. 2016; [PubMed: 26861699]

44. Qi Y, et al. A brush-polymer/exendin-4 conjugate reduces blood glucose levels for up to five days and eliminates poly(ethylene glycol) antigenicity. Nat Biomed Eng. 1: 0002. 2017;

45. Hsu T, Mitragotri S. Delivery of siRNA and other macromolecules into skin and cells using a peptide enhancer. Proc Natl Acad Sci USA. 108: 15816-15821. 2011. [PubMed: 21903933]

46. Yao YD, et al. Targeted delivery of PLK1-siRNA by ScFv suppresses Her2+ breast cancer growth and metastasis. Sci Transl Med. 4: 130ra148. 2012;

47. Chen Q, et al. Lipophilic siRNAs mediate efficient gene silencing in oligodendrocytes with direct CNS delivery. J Control Release. 144: 227-232. 2010. [PubMed: 20170694]

48. Singh N, Agrawal A, Leung AK, Sharp PA, Bhatia SN. Effect of nanoparticle conjugation on gene silencing by RNA interference. J Am Chem Soc. 132: 8241-8243. 2010. [PubMed: 20518524]

49. Breunig M, et al. Mechanistic investigation of poly(ethylene imine)-based siRNA delivery: disulfide bonds boost intracellular release of the cargo. J Control Release. 130: 57-63. 2008. [PubMed: 18599144]

50. Potera C. Antisense-down, but not out. Nat Biotechnol. 25: 497-499. 2007. [PubMed: 17483826]

51. Studier FW. Protein production by auto-induction in high density shaking cultures. Protein Expr Purif. 41: 207-234. 2005. [PubMed: 15915565] 
52. Kim CH, et al. Bispecific small molecule-antibody conjugate targeting prostate cancer. Proc Natl Acad Sci USA. 110: 17796-17801. 2013. [PubMed: 24127589]

53. Stratford S, et al. Examination of real-time polymerase chain reaction methods for the detection and quantification of modified siRNA. Anal Biochem. 379: 96-104. 2008. [PubMed: 18501185]

54. Eguchi A, et al. Efficient siRNA delivery into primary cells by a peptide transduction domaindsRNA binding domain fusion protein. Nat Biotechnol. 27: 567-571. 2009. [PubMed: 19448630]

55. Beck J, et al. Ubiquitylation-dependent localization of PLK1 in mitosis. Nat Cell Biol. 15: 430439. 2013. [PubMed: 23455478]

56. Höbel, S, Aigner, A. RNA Interference: From Biology to Clinical Applications. Min, W-P, Ichim, T, editors. Humana Press; Totowa, NJ: 2010. 283-297. 


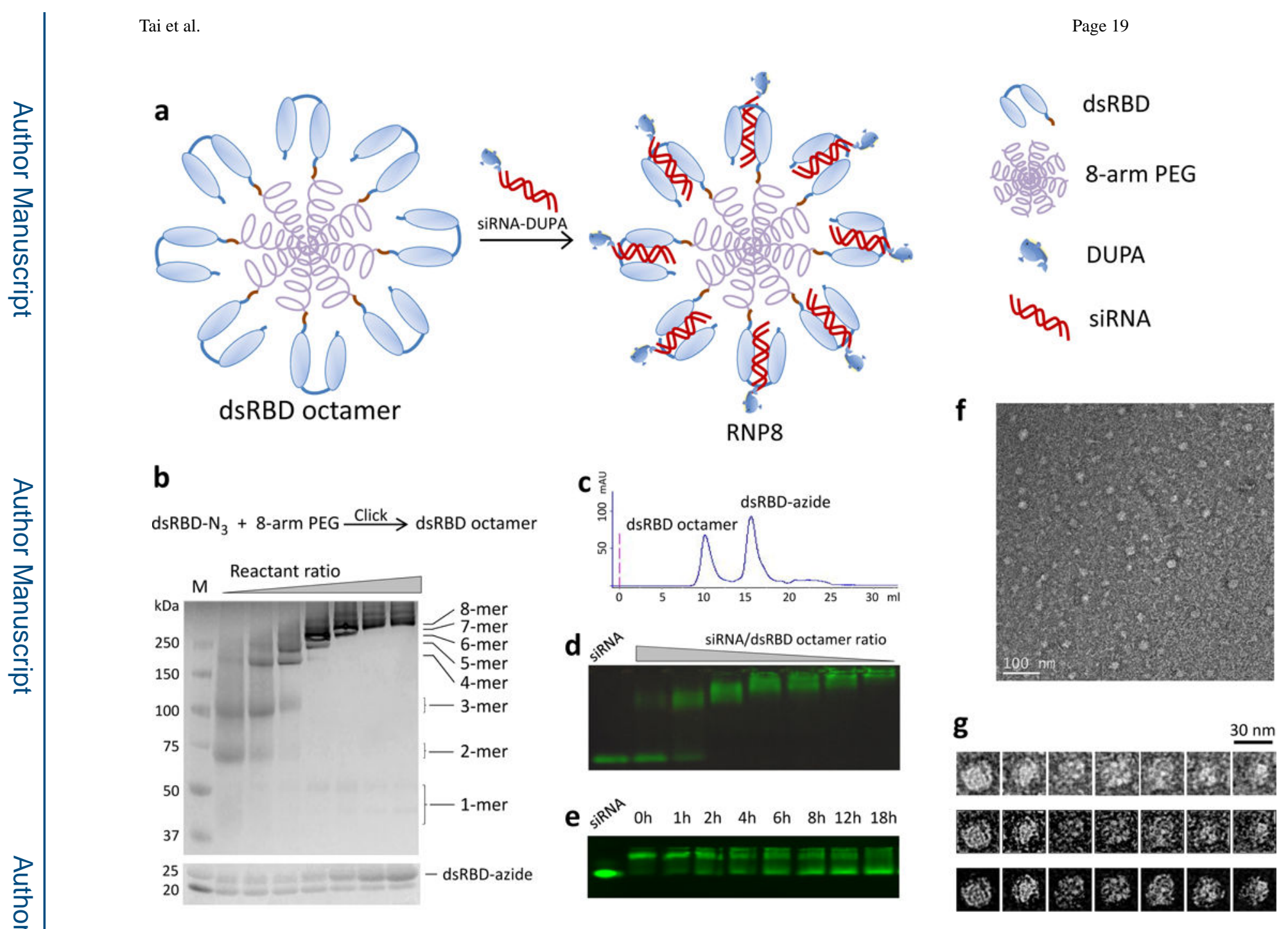

Figure 1. Schematics and characterizations of $\mathrm{RNP}_{8}$ and its intermediates

(a) Assembly between multivalent dsRBD and siRNA-targeting ligand bioconjugates, resulting in cargo loading with precisely controlled stoichiometry and highly accessible targeting ligands. (b) Oligomerization of dsRBD-azide on 8-arm DBCO-PEG via copperfree click chemistry. SDS-PAGE gel electrophoresis indicates that increasing molar ratio of dsRBD over PEG eventually leads to saturated PEGylation (formation of octamer). (c) Separation of dsRBD octamer from excess dsRBD using Superdex ${ }^{\mathrm{TM}} 200$ size exclusion chromatography. (d) Titration of varying amount of dsRBD octamer to siRNA of fixed quantity. 20 pmol of FAM-labeled siRNA is titrated with varying amount of dsRBD octamer (from left to right: $0,1.25,2.5,5,10,15,20$ and 40 pmol. $\mathrm{RNP}_{8}$ is obtained at molar ratio 16/1 and 8/1 ( $2^{\text {nd }}$ and $3^{\text {rd }}$ lanes from the left). More dsRBD octamer leads to unsaturated octamer with smaller gel shifts $\left(4^{\text {th }}-8^{\text {th }}\right.$ lanes $)$. Additional fine titration studies near ratio 8 can be found in Supplementary Figure S4. (e) Stability of $\mathrm{RNP}_{8}$ in $50 \%$ mouse serum at $37^{\circ} \mathrm{C}$. Dissociated siRNA is quantified against the pure siRNA band $\left(1^{\text {st }}\right.$ lane from the left). Quantitative dot plot of the gel band fluorescence intensities is shown in Supplementary Figure S5. The dissociation is slow with a half-life of approximately $18 \mathrm{~h}$. (f) TEM micrographs of $\mathrm{RNP}_{8}$ stained by uranyl formate showing uniform and compact 
nanoparticles. (g) Selected raw images of 7 representative $\mathrm{RNP}_{8}$ particles. Raw images are shown in the upper row, and the corresponding low-pass filter processed and masked noise-reduced images are shown in the middle row and bottom row, respectively. 


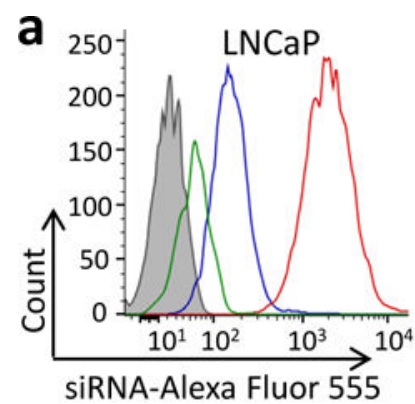

b
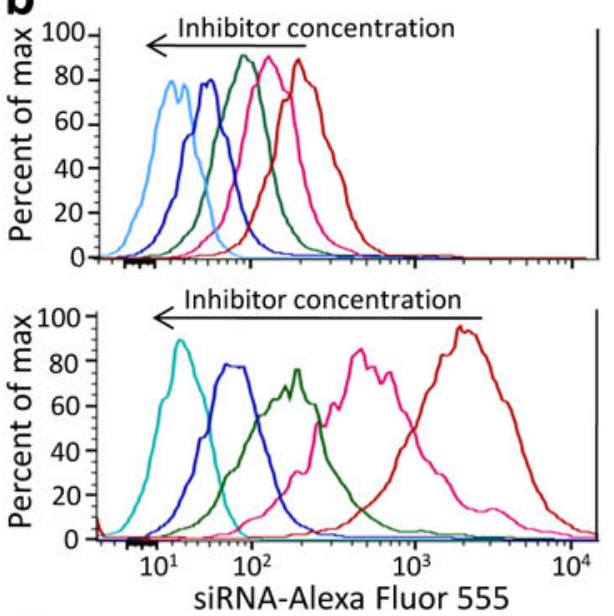

d

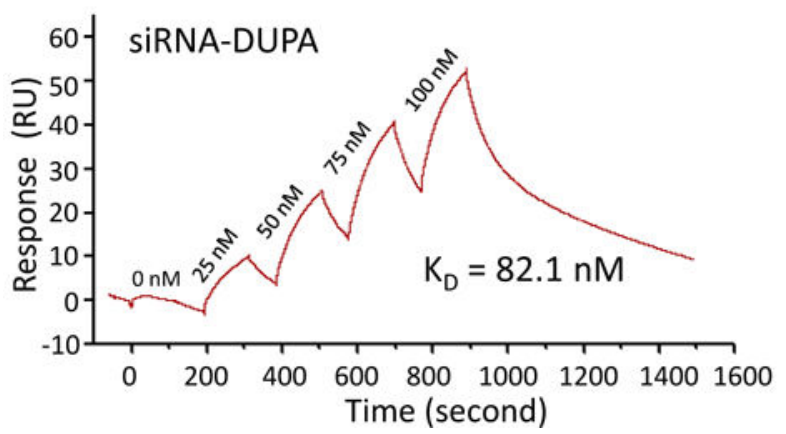

C
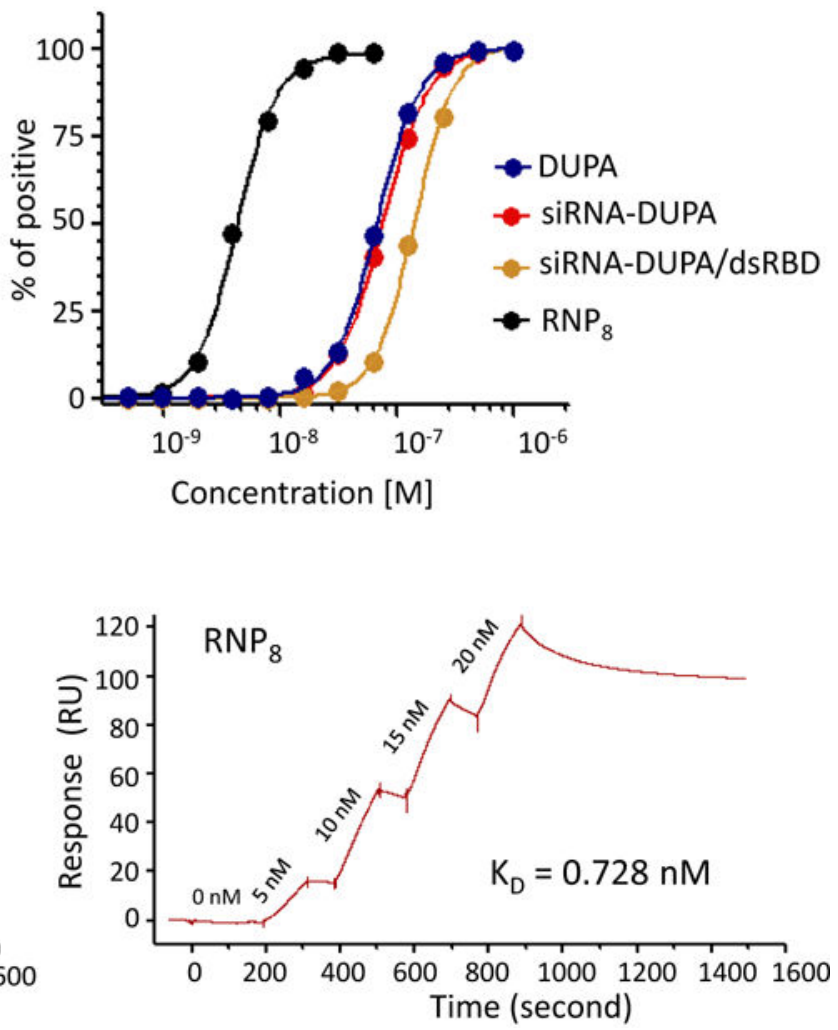

Figure 2. PSMA-mediated specific RNP8 binding to cells

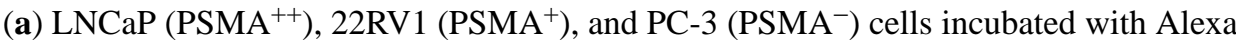
Fluor 555-labeled siRNA-DUPA or $\mathrm{RNP}_{8}$ (the RNA component is also labeled with the same fluorophore) at RT for $1 \mathrm{~h}$. Cell binding is assessed by flow cytometry, after cells are treated with $\mathrm{RNP}_{8}$ or siRNA-DUPA (siRNA concentrations for both experiments are $100 \mathrm{nM}$ ). (b) Equilibrium competition binding assayby addition of PMPA to siRNA-DUPA treated $\mathrm{LNCaP}$ cells (upper panel) and $\mathrm{RNP}_{8}$ treated $\mathrm{LNCaP}$ cells (bottom panel). The siRNA concentrations for both experiments are $100 \mathrm{nM}$. The inhibitor concentration (from left to right) are 2,000 nM, $400 \mathrm{nM}, 50 \mathrm{nM}, 0 \mathrm{nM}$ (4 curves on the right) for the upper panel, in comparison with untreated cells (curve on the left); 10,000 nM, $800 \mathrm{nM}, 100$ $\mathrm{nM}, 0 \mathrm{nM}$ (4 curves on the right) for the bottom panel, in comparison with untreated cells (curve on the left). (c) Profiling the binding affinity of Alexa Fluor 555-labeled DUPA, 
siRNA-DUPA, complex of siRNA-DUPA and dsRBD monomer, and $\mathrm{RNP}_{8}$ to $\mathrm{LNCaP}$ cells. $\mathrm{RNP}_{8}$ shows higher binding affinity than siRNA-DUPA, than siRNA-DUPA complexed with dsRBD monomer. (d) Biacore SPR analysis of the dissociation constant $\left(\mathrm{K}_{\mathrm{D}}\right)$ between siRNA-DUPA and PSMA, and between $\mathrm{RNP}_{8}$ and PSMA. $\mathrm{RNP}_{8}$ shows significantly higher binding affinity (lower $\mathrm{K}_{\mathrm{D}}$ ) to the PSMA receptor compared to the monovalent siRNADUPA. 


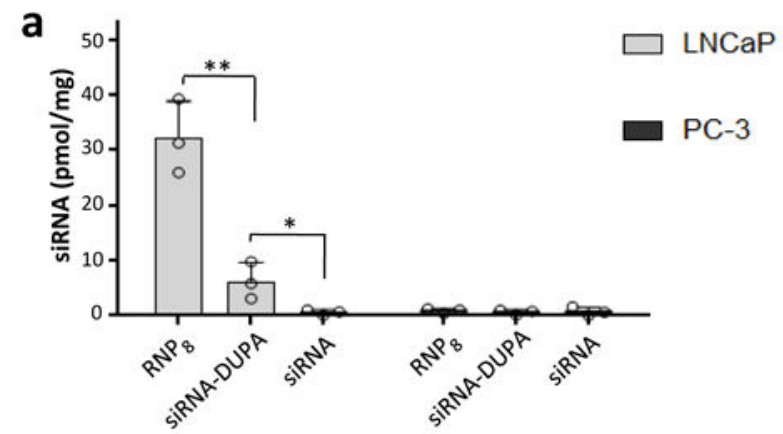

C $\mathrm{RNP}_{8}$ (truncated)
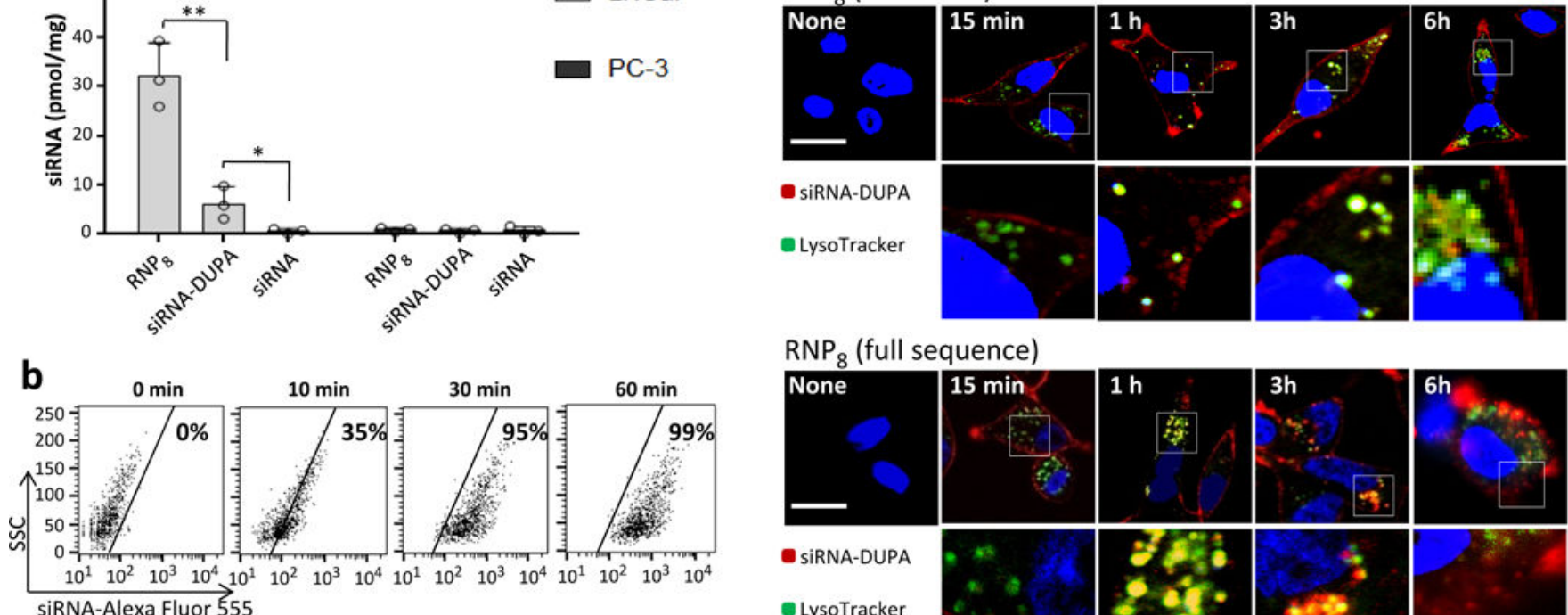

- siRNA-DUPA

- LysoTracker

$\mathrm{RNP}_{8}$ (full sequence)
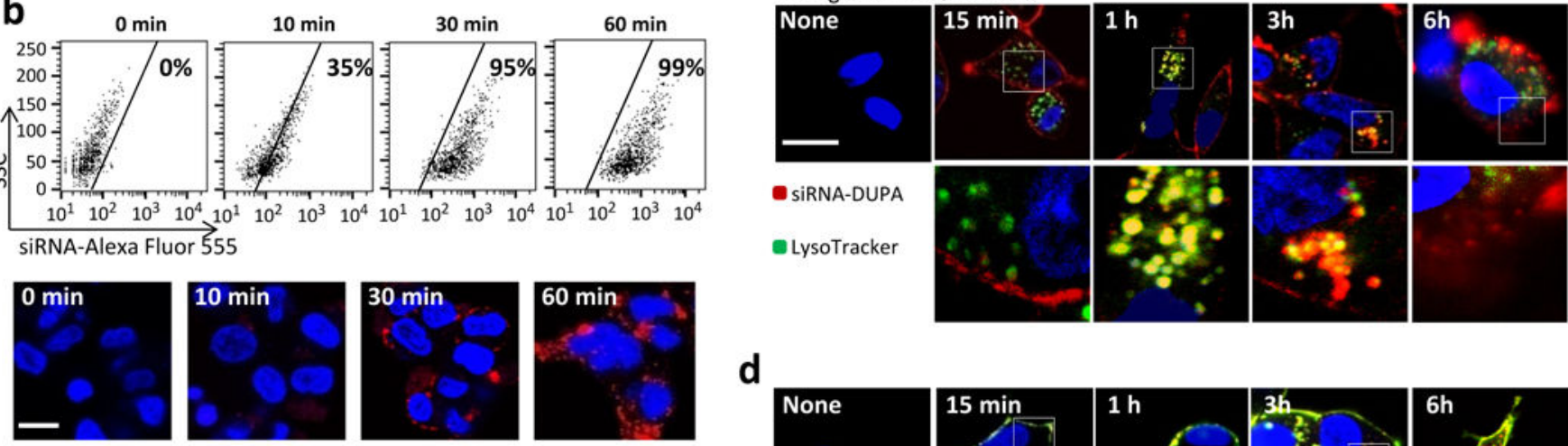

- siRNA-DUPA

- LysoTracker
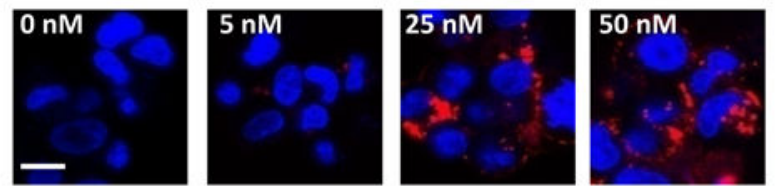

e

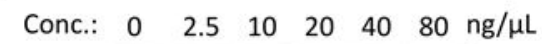

Total RNA

miRNA

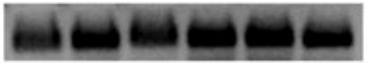

TAR RNA
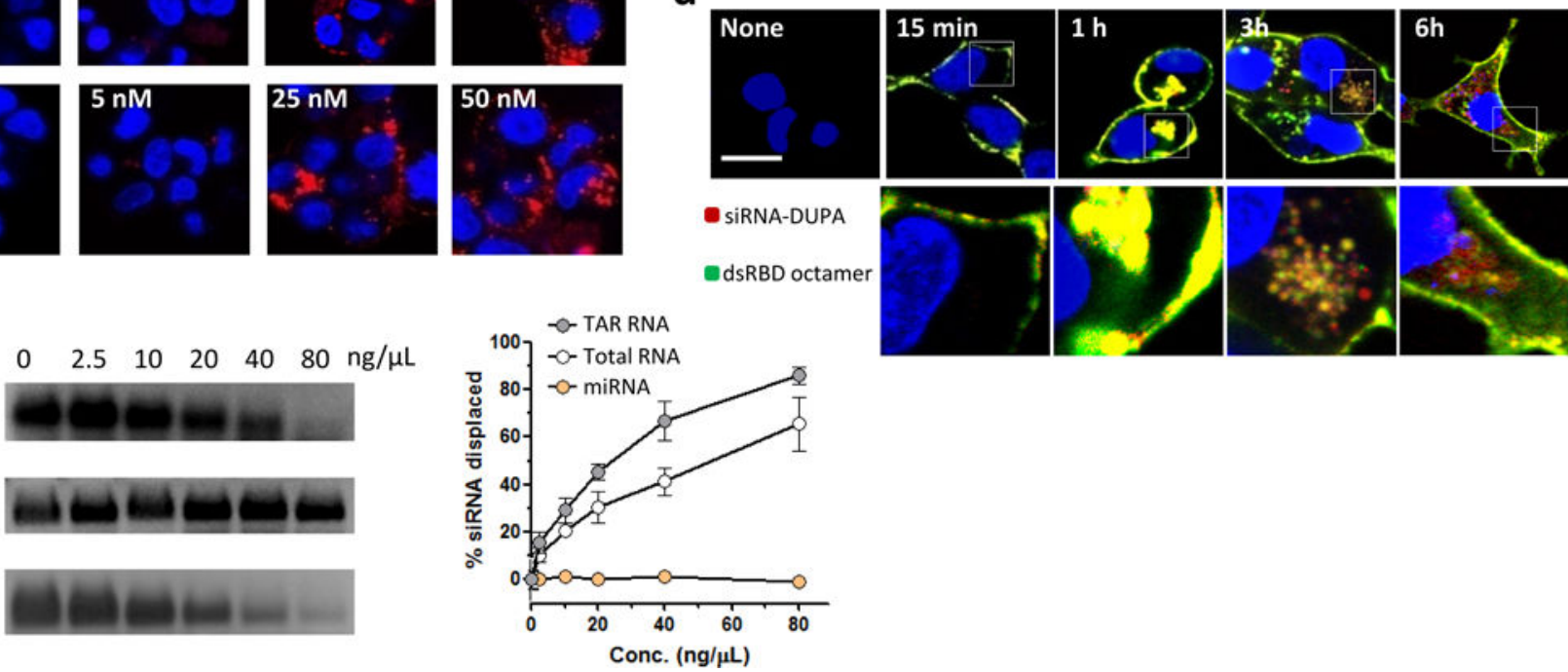

Figure 3. $\mathrm{RNP}_{8}$ cell uptake and intracellular trafficking

(a) Quantification of siRNA uptake using stem-loop real-time PCR. siRNA uptake after $2 \mathrm{~h}$ incubation at $37^{\circ} \mathrm{C}$ is normalized to the concentration of total protein in cell lysis (mean \pm s.d., $\mathrm{n}=3$ ). ${ }^{*}, p=0.0461 ; * *, p=0.0039$ (two-tailed t-test). (b) Kinetics of $\mathrm{RNP}_{8}$ internalization into LNCaP cells. After acid-wash of the surface-bound $\mathrm{RNP}_{8}$, the internalized siRNA is analyzed by flow cytometry (top row) and confocal microscopy (middle and bottom rows). For time dependent uptake, LNCaP cells are treated with $\mathrm{RNP}_{8}$ (total siRNA-DUPA concentration $50 \mathrm{nM}$, fluorescence labeled). For concentration dependent uptake, the incubation time is $1 \mathrm{~h}$. Scale bar, $10 \mu \mathrm{m}$. (c) Intracellular trafficking of truncated $\mathrm{RNP}_{8}$ (without the $\mathrm{H}_{2}$ E repeats) and $\mathrm{RNP}_{8}$ (full sequence) in LNCaP cells. siRNA-DUPA is labeled with Alexa Fluor 555 (red) and endosome/lysosome is labeled with LysoTracker Green (green). Appearance of intracellular red fluorescence for $\mathrm{RNP}_{8}$ 
(full sequence) after 6-h incubation indicates endosomal escape (low-magnification image shown in Supplementary Figure S8). The truncated $\mathrm{RNP}_{8}$ only shows some red fluoresce on cell surface due to initial binding, but virtually no red signal inside cells. Scale bar, $10 \mu \mathrm{m}$. (d) Intracellular monitoring of $\mathrm{RNP}_{8}$ dissociation in LNCaP cells. siRNA-DUPA (Alexa Fluor 555, red) remains associated with dsRBD octamer (Alexa Fluor 488, green) on the cell membrane and in early-stage endosomes (merged color yellow), while dissociating beyond $3 \mathrm{~h}$ incubation. Scale bar, $10 \mu \mathrm{m}$. (e) Dose-dependent siRNA displacement from ribonucleoprotein (dsRBD-siRNA complex) by total RNA isolated from cells, miRNA, or TAR RNA (the curves are plotted as means \pm s.d., $n=3$ ). The TAR RNA is the natural binding substrate of dsRBD and serves as a positive control in this experiment. The full images are displayed in Supplementary Figure S10. 
a

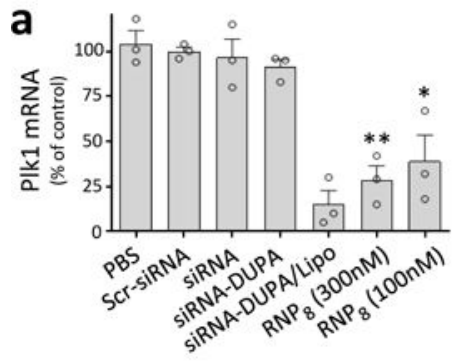

e
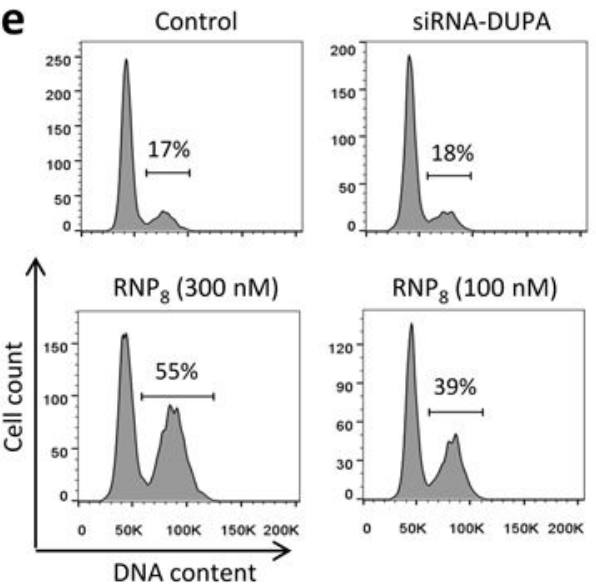

b

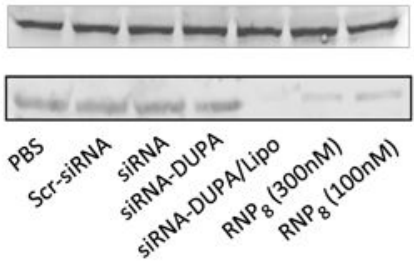

C

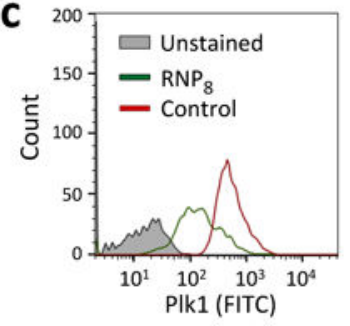

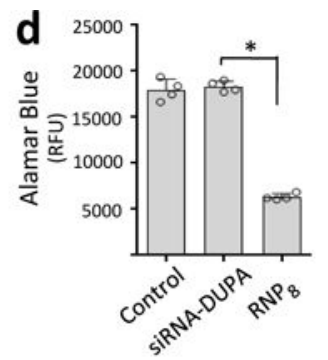

f
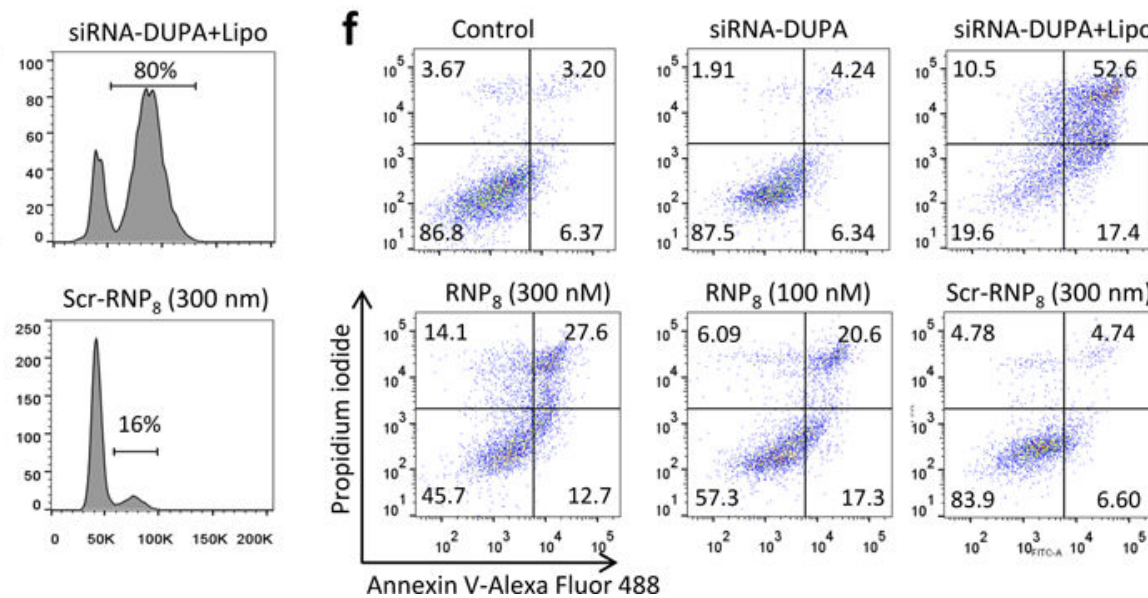

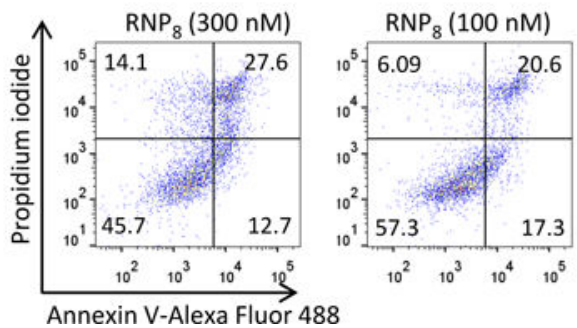

Annexin V-Alexa Fluor 488

\section{.}

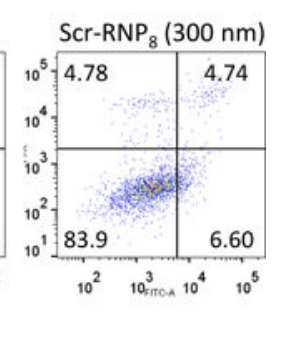

Figure 4. Silencing of Plk1 by RNP8 in vitro

(a) Gene silencing by $\mathrm{RNP}_{8}$ compared to controls including scrambled control siRNA, siRNA only, siRNA-DUPA only, and siRNA-DUPA in the presence of Lipofectamine 2000. RNAi is quantified by real-time PCR (mean \pm s.e.m., $\mathrm{n}=3$ ). $p=0.0144 ; * *, p=$ 0.0009 compared to the scrambled control (two-tailed t-test). (b) Plk1 protein expression determined by Western blotting. Full images are available in Supplementary Figure S11. (c) Plk1 protein expression assessed by flow cytometry. (d) Proliferation of LNCaP cells treated with siRNA-DUPA or $\mathrm{RNP}_{8}$ (determined with the Alamar Blue assay, mean \pm s.d., $\mathrm{n}=4$ ). RFU, relative fluorescence unit. *, $p=0.0001$ (two-tailed t-test). (e) Cell cycle profiles of $\mathrm{LNCaP}$ cells treated by various siRNA agents. The DNA content is determined by flow cytometry $48 \mathrm{~h}$ post treatment. siRNA-DUPA transfected with Lipofectamine is used as a positive control for mitotic arrest. Scr-RNP ${ }_{8}$ : $\mathrm{RNP}_{8}$ with scrambled control siRNA. (f) Cell apoptosis determined by Annexin V and PI staining. Viable, early apoptotic, late apoptotic and necrotic cell populations (\%) are shown in the lower left, lower right, upper right, and upper left quadrants, respectively. Results are representatives of three independent experiments obtained with flow cytometry. 

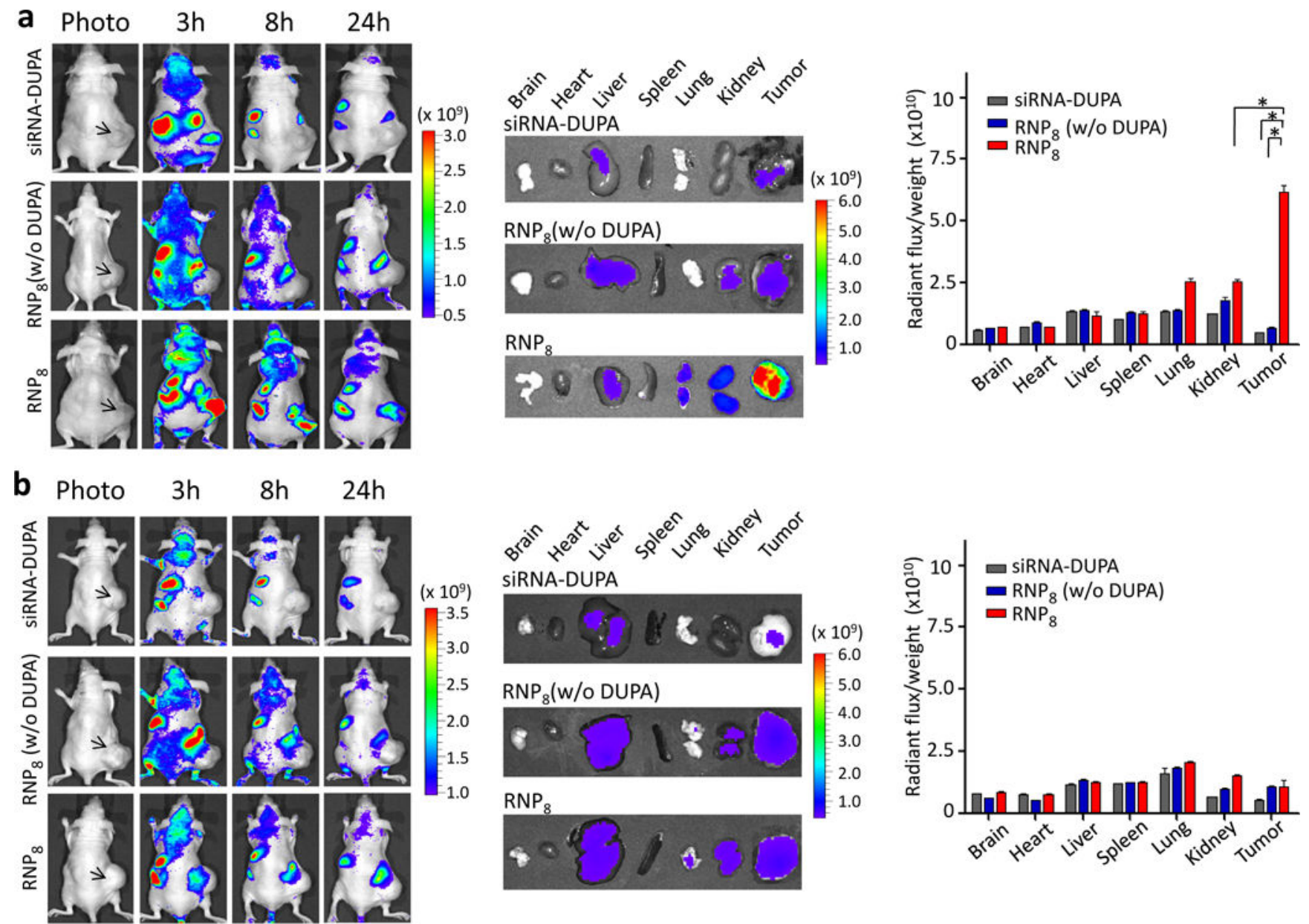

\section{d}

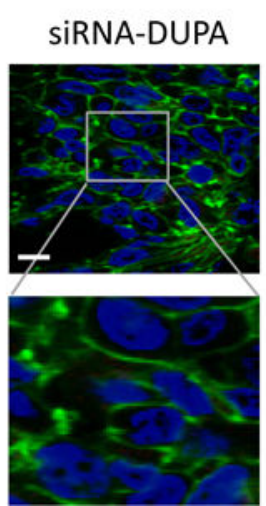

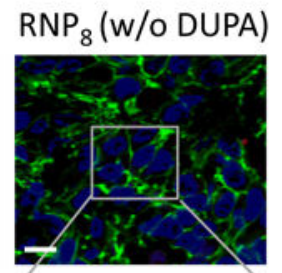

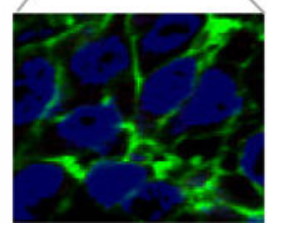

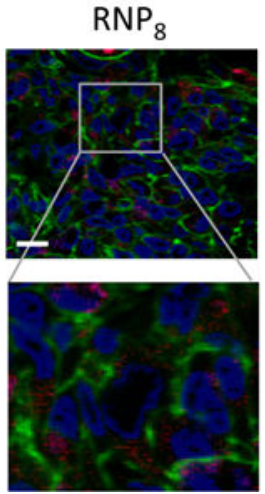

Figure 5. Biodistribution of siRNA-DUPA and RNP8 in tumor bearing mice

(a \& b) NIRF imaging of mice bearing LNCaP or PC-3 tumors after intravenous injection of Alexa Fluor 680-labeled siRNA-DUPA, $\mathrm{RNP}_{8}$ without DUPA or $\mathrm{RNP}_{8}$. Left column: whole-body NIRF imaging of mice. The black arrow indicates the location of tumor (the sizes of the tumors shown are 442, 540, 464, 480, 588, and $560 \mathrm{~mm}^{3}$, respectively). Middle column: ex vivo imaging of tumors and other major organs $24 \mathrm{~h}$ post injection. Right: quantitative analysis of the ex vivo fluorescence signals (mean \pm s.d., $\mathrm{n}=3$ ). *,$p<0.05$ (two-tailed t-test). (c) Pharmacokinetic study of siRNA-DUPA and $\mathrm{RNP}_{8}$ by quantifying 
blood siRNA concentration in mice using stem-loop real-time PCR (mean \pm s.d., $\mathrm{n}=3$ ). (d) Confocal microscopic images of tumor sections from mice injected intravenously with siRNA-DUPA, $\mathrm{RNP}_{8}$ w/o DUPA, or $\mathrm{RNP}_{8}$. Red, Alexa Fluor 555-labeled siRNA; green, Alexa Fluor 488 Phalloidin; blue, nuclear staining with DAPI. Scale bar, $10 \mu \mathrm{m}$. 
a

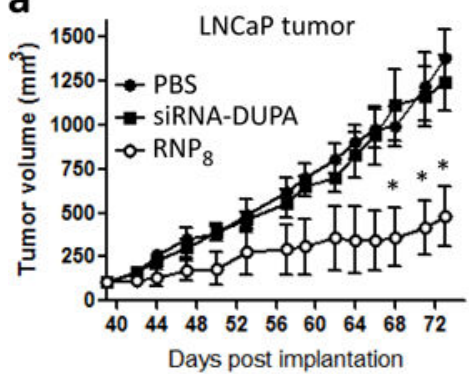

C

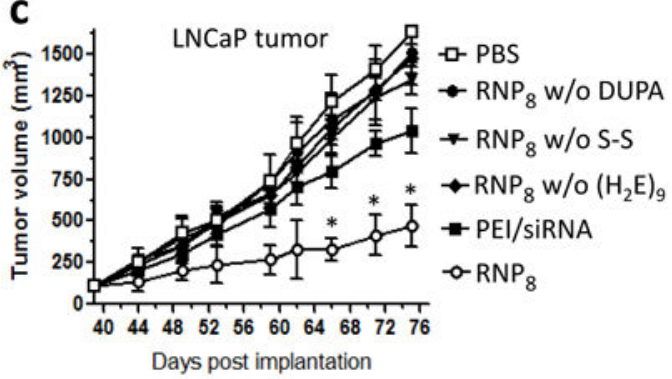

d
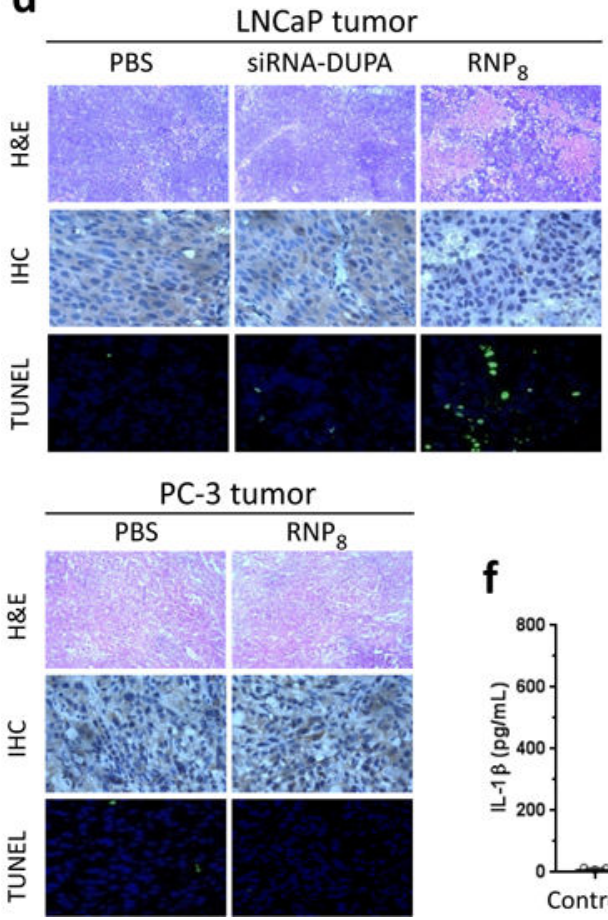

f

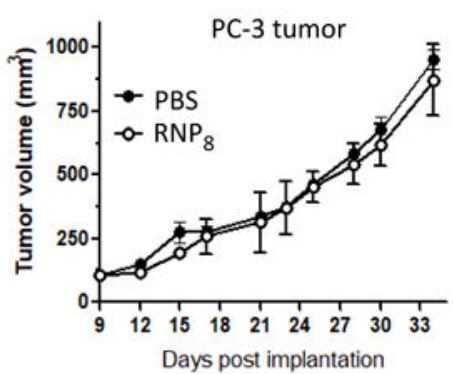

e $\left.\quad \mathrm{IL}-1 \beta(\mathrm{pg} \mathrm{ml})^{-1}\right)$

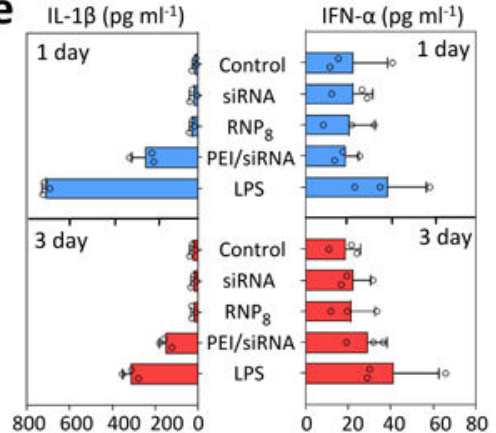

IFN- $\gamma\left(\mathrm{pg} \mathrm{ml}^{-1}\right)$
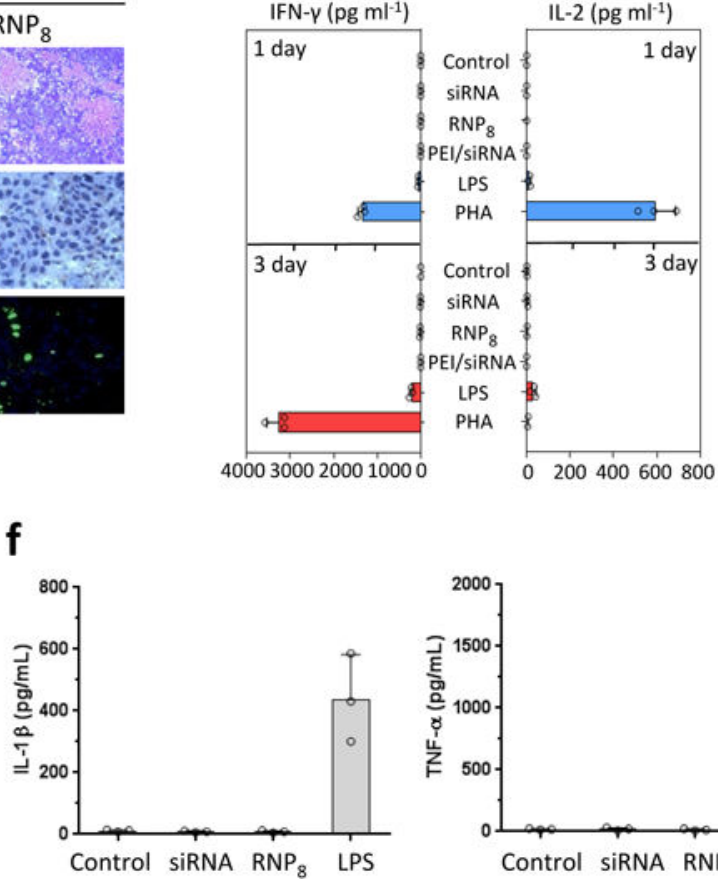

b

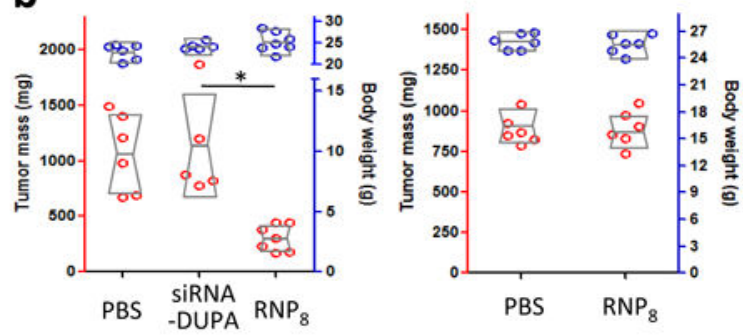

Figure 6. In vivo RNAi with RNP8

(a) Mice bearing LNCaP tumors (left panel) and PC-3 tumors (right panel) were injected with PBS, siRNA-DUPA or $\mathrm{RNP}_{8}$ intravenously at equivalent siRNA dose $(2 \mathrm{mg} / \mathrm{kg})$ every third day, starting on the $39^{\text {th }}$ day post $\mathrm{LNCaP}$ implantation or $9^{\text {th }}$ day post PC-3 implantation (mean \pm s.e.m., $\mathrm{n}=5-7$ ) *, $p<0.05$ (two-tailed t-test). (b) Grouped scatterplots showing the tumor mass (red dots against y-axis on the left) and body weight (blue dots against $\mathrm{y}$-axis on the right) at the endpoint of the treatment (mean \pm s.d., $\mathrm{n}=5-7)$. ${ }^{*}, p=$ 0.0011 (two-tailed t-test). (c) Antitumor treatment comparison of PBS, PEI/siRNA, RNP , 
and a number of $\mathrm{RNP}_{8}$ missing certain functionalities in mice bearing $\mathrm{LNCaP}$ tumors (mean \pm s.e.m., $\mathrm{n}=5$-6). ${ }^{*}, p<0.05$ compared to PBS (two-tailed t-test). (d) Histological study showing a serpiginous area of necrosis (H\&E staining), reduced Plk1 protein expression (IHC staining), and cell apoptosis (TUNEL staining) in LNCaP tumors treated with $\mathrm{RNP}_{8}$ compared to control groups treated with PBS or siRNA-DUPA (upper panels), whereas virtually no difference is observed between $\mathrm{RNP}_{8}$ treated PC-3 tumor and controls (bottom panels). (e) Analysis of cytokine induction in human PBMCs at 1 day or 3 days after treatment with PBS, siRNA ( $2^{\prime} \mathrm{OMe}$ ), $\mathrm{RNP}_{8}$, or siRNA plus PEI. Lipopolysaccharide (LPS, $10 \mu \mathrm{g} / \mathrm{mL}$ ) and phytohemagglutinin (PHA, $20 \mu \mathrm{g} / \mathrm{mL}$ ) are used as positive controls. Cytokines that regulate the innate immunity, IL- $1 \beta$, IFN- $\alpha$, TNF- $\alpha$, and IL-6, are shown in the upper row, and cytokines that regulate adaptive immunity, IFN- $\gamma$, IL-2, IL-4 and IL-13, are shown in the bottom row (mean \pm s.d., $\mathrm{n}=3$ ). (f) Analysis of early innate cytokine induction by PBS, siRNA ( $2^{\prime} \mathrm{OMe}$ ), $\mathrm{RNP}_{8}$ and LPS in C57BL/6 mice (mean \pm s.d., $\mathrm{n}=3$ ). 